\title{
Estudio florístico de la porción central del Municipio de San Jerónimo Coatlán, Distrito de Miahuatlán (Oaxaca)
}

\author{
ÁLVARO CAMPOS-VILLANUEVA Y JOSÉ LUIS VILLASEÑOR
}

Instituto de Biología, UNAM, Apdo. Postal 70-367, 04510, México, D. F.

\begin{abstract}
Resumen. Este trabajo es una contribución al conocimiento florístico de la Sierra Madre del Sur, una de las regiones, desde el punto de vista botánico, menos exploradas del estado de Oaxaca. El estudio se llevó a cabo en la porción central del Municipio de San Jerónimo Coatlán, Distrito de Miahuatlán. La flora fanerogámica de la región incluye 123 familias, 370 géneros y 680 especies. Se hace una breve descripción de los tres tipos de vegetación presentes en la zona de estudio, así como una discusión de las afinidades de la flora con otras regiones de México.

Palabras clave: fanerógamas, florística, gimnospermas, magnoliophyta, México, Oaxaca.

Abstract. This paper is a contribution to a better knowledge of the Sierra Madre del Sur, one of regions in Oaxaca less known and studied floristically. The study was carried out in the central portion of Municipality of San Jerónimo Coatlán, District of Miahuatlán. The phanerogamic flora of the region includes 123 families, 370 genera and 680 species. Short descriptions of the three vegetation types found in the area are provided, and the floristic affinities with other Mexican regions are discussed.
\end{abstract}

Keywords: phanerogams, floristics, gymnosperms, magnoliophyta, Mexico, Oaxaca.

\section{INTRODUCCIÓN}

La exploración botánica en el estado de Oaxaca se inició a finales del siglo XVIII, cuando la expedición botánica a la Nueva España, encabezada por Martín Sessè, llegó a Tehuantepec (Dávila y Germán, 1991). Desde entonces, un gran número de botánicos y naturalistas ha llevado a cabo exploraciones en el estado, entre los que se encuentran A. von Humboldt, A. F. Liebmann, C. G. Pringle, C. Conzatti, T. MacDougall y F. Miranda, por mencionar algunos. Sus aportaciones fueron la base del conocimiento florístico del estado hasta mediados del presente siglo.

En tiempos recientes, se iniciaron trabajos más sistemáticos de colecta botánica por el personal del Herbario Nacional (MEXU) del Instituto de Biología de la UNAM (Lorence y García, 1989). El programa de exploración botánica continúa hasta ahora, dedicado principalmente a la colecta intensiva y exhaustiva en zonas escogidas. Aunque se ha colectado en gran parte del estado y no obstante que se llevan muchos años de colecta florística, hay muchas áreas que no han sido exploradas o, en su defecto, sólo hay informes muy superficiales que no dan una visión real de la flora. Una de las regiones en tal situación es la Sierra Madre del Sur, la cual, a pesar de haber sido visitada y colectada desde el siglo XIX, nunca se ha estudiado detalladamente.

Antes de la realización de este estudio, las colectas conocidas para la Sierra Madre del Sur correspondientes al proyecto Flora de Oaxaca, llevado a cabo por personal del Herbario Nacional, se estimaban en 4,260 números, de los cuales aproximadamente 350 eran del Municipio de San Jerónimo Coatlán (García y Torres, en prensa). Aunque han pasado algunos colectores por la región, en realidad no se ha hecho ningún estudio detallado de ella. El presente trabajo intenta cubrir esta carencia, contribuyendo al conocimiento de una porción de esta sierra.

El Municipio de San Jerónimo Coatlán jamás fue visitado por botánicos que han contribuido significativamente a la flora del estado (por ejemplo, C. Conzatti,F. M.Liebmann o T. MacDougall) aunque algunos de ellos llegaron a estar relativamente cerca. Los reportes publicados para la Sierra Madre del Sur son muy generales y poco descriptivos de la vegetación, aunque hacen énfasis en la riqueza florística de la región (Conzatti, 1918, 1922, 1929). Los objetivos de este trabajo son elaborar un listado de la flora fanerogámica encontrada en la porción central del Municipio de San Jerónimo Coatlán, Distrito de Miahuatlán, describir la vegetación con base en su fisonomía y composición florística, y discutir las relaciones de esta flora con algunas otras regiones de México.

\section{DESCRIPCIÓN DEL ÁREA DE ESTUDIO}

La zona de estudio se ubica en la porción central del municipio de San Jerónimo Coatlán, Distrito de Miahuatlán, aproximadamente a $70 \mathrm{~km}$ al SO de la ciudad de Miahuatlán y a $50 \mathrm{~km}$ al SE del poblado de Juchatengo (Fig. 1). Las vías de acceso son tres: por el occidente, a partir de Miahuatlán, de donde sale la brecha hacia los Coatlanes; del SE se llega a partir de la carretera Sola de Vega-Oaxaca por la desviación hacia Soledad Piedra Larga y San Jerónimo Coatlán, y del NO se puede llegar por la brecha que viene de Juchatengo hacia los Coatlanes, pasando por Cruz de Honduras (Fig. 1). La zona comprende una superficie aproximada de 17,300 hectáreas y se ubica entre los paralelos $16^{\circ} 09^{\prime}$ y $16^{\circ} 15^{\prime}$ de latitud $\mathrm{N}$ y los meridianos $96^{\circ} 52^{\prime}$ y $97^{\circ} 01^{\prime}$ de longitud $\mathrm{O}$. 


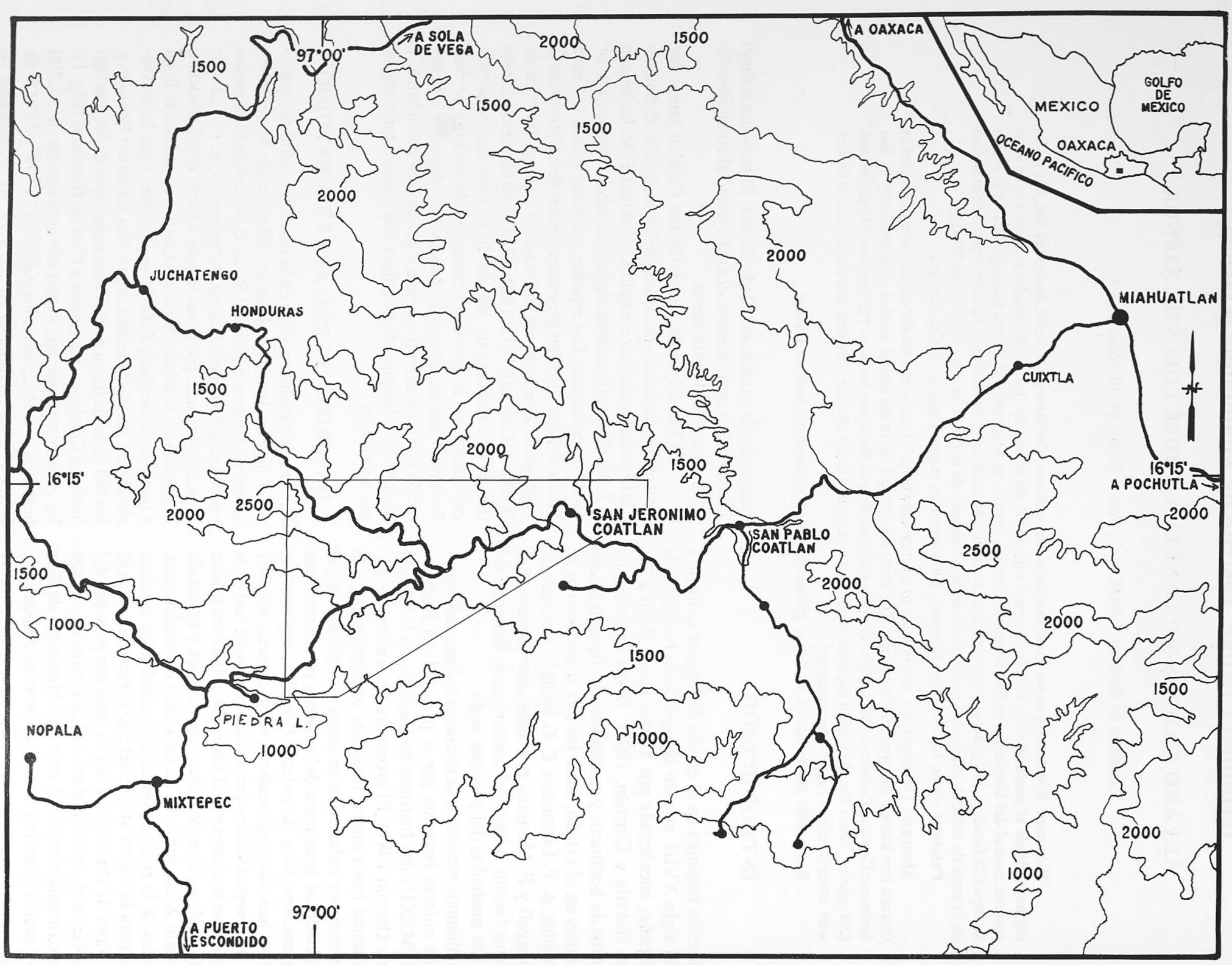

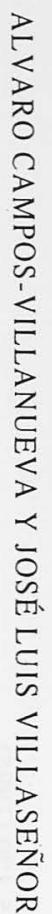


El origen y fundación de San Jerónimo Coatlán se remonta a1612 (Secretaría de Gobernación y Gobierno del Estado de Oaxaca, 1988) y es de raíces zapotecas. Sus pobladores originalmente vivían de la agricultura y caza. El nombre de Coatlán significa «junto a, lugar de las culebras» y deriva de las raíces en Náhuatl Coatl= culebra y Tlan= junto a, lugar.

El área está comprendida dentro de la provincia fisiográfica denominada Sierra Madre del Sur (Rzedowski, 1978), caracterizada por una topografía muy accidentada y altitudes que van de $\operatorname{los} 1,000$ a $\operatorname{los} 2,700 \mathrm{msnm}$. En la zona se encuentra una gran cantidad de barrancas y cañadas con riachuelos, la mayoría de ellos permanentes, que originan microhábitats muy particulares.

La zona de estudio se halla en el parteaguas de la Sierra Madre del Sur, donde se encuentra, por un lado, la vertiente del Pacífico, con un alto porcentaje de humedad y bosques densos y, por el otro, la conexión hacia el valle de Miahuatlán y los valles centrales de Oaxaca, con climas más secos y bosques menos densos, que presentan una marcada estacionalidad.

De acuerdo con López-Ramos (1974), esta región pertenece a la provincia geológica de la Sierra Madre del Sur. Los afloramientos existentes son principalmente de rocas metamórficas del Jurásico, formados por esquistos y gneiss, con intrusiones de rocas graníticas. También está incluida una pequeña porción de origen precámbrico al $\mathrm{N}$ NE de la zona de estudio.

Las rocas jurásicas son un grupo de diversa litología, dentro del denominado «Complejo Xolapa» (INEGI, 1988c), cuyo metamorfismo se origina por las altas presiones y temperaturas ocasionadas por la convergencia de placas en la zona orogénica circunpacífica. La asociación litológica incluye gneiss, cuarzo feldespático y esquisto. Es común en lazona encontrar bandeamiento, micropliegues e intemperismo hasta de 2 y $3 \mathrm{~m}$ de profundidad.

Las rocas precámbricas, denominadas «Complejo Oaxaqueño» consisten en gneiss y cuarzo feldespático. Subyacen discordantemente a las calizas de la formación Teposcolula, así como a la unidad Tom. El INEGI (1988c) indica también una relación de contacto tectónico con las rocas del complejo Xolapa.

El suelo de la zona de estudio es un nitrosol dístrico, típico de una zona muy lluviosa cálido-templada (SARH, 1985), que se caracteriza por presentar una capa arcillosa de color rojizo, muy profunda, algunas veces de hasta 5-10 m, cubierta por otra de grosor considerable de materia orgánica. Esto se cumple para casi toda la zona, excepto en la porción N-NE, donde se presenta como un suelo calizo, poco profundo y pobre en materia orgánica. En algúnas áreas se observan pequeños afloramientos de roca madre.

Para poder dar una clasificación del clima en la región, se usó la carta de efectos climáticos 1: 250,000 (INEGI, 1985), ya que en la zona no hay ninguna estación climatológica. Las medias anuales de precipitación, para la zona en general, oscilan entre 700 y $1,500 \mathrm{~mm}$, con una temperatura media anual de 18 a $22^{\circ} \mathrm{C}$ (INEGI, 1988d). La época seca comprende de noviembre a abril, y la húmeda abarca de mayo a octubre.

La precipitación total durante la temporada de secas fluctúa entre 75 y $125 \mathrm{~mm}$, con temperaturas mínimas de 6 a $12^{\circ} \mathrm{C}$ y máximas de 21 a $27^{\circ} \mathrm{C}$. En la época de lluvias, la precipitación aumenta considerablemente, ya que llueve entre 800-900 mm en las áreas más «secas» y 900-1,700 mm en las de mayor humedad.

Tomando como base la misma carta (INEGI, 1985) se pudieron inferir los tipos de climas presentes en la región, de acuerdo con el sistema de clasificación de Köppen modificado por García (1981); éstos son:

AW2=Cálido subhúmedo. Se ubica al S-SO de la zona, entre los 1,000-1,300 msnm, y es el más cálido de todos.

$\mathrm{A}(\mathrm{C}) \mathrm{m}(\mathrm{W})=$ Semicálido húmedo. Es el que abarca la mayor parte de la zona de estudio, entre los 1,300 y 2,100 msnm, y se caracteriza por presentar la mayor cantidad de precipitación.

$\mathrm{A}(\mathrm{C}) \mathrm{W} 1(\mathrm{~W})=$ Semicálido subhúmedo intermedio. Este tipo de clima se restringe a una pequeña porción al $\mathrm{N}$ NE del área, entre los 1,400 y 1,700 msnm; es el más seco de los cálidos, así como el de las temperaturas más extremosas del área.

C(W2) = Templado subhúmedo. Confinado a los picos y partes más altas de la región, es donde se registran las temperaturas más bajas. Se presenta entre los 2,100 y 2,500 msnm, y también se caracteriza por una buena cantidad de precipitación.

$\mathrm{C}(\mathrm{W} 1)(\mathrm{W})=$ Templado subhúmedo intermedio. Se presenta en la parte $\mathrm{N}$ del área de estudio, entre los 1,800 y $2,100 \mathrm{msnm}$.

Como la región de estudio está comprendida en el parteaguas de la Sierra Madre del Sur, existen dos cuencas por donde drenan las montañas del área. En la vertiente SSO existe una gran cantidad de arroyos permanentes y semipermanentes surcando todas las cañadas y barrancas de esta serranía, las cuales más adelante se unen para dar lugar a los ríos La Rana, Leche, La Piedra Ancha, y Ballesteros. Todos estos arroyos son tributarios del Río Colotepec, que finalmente llega a depositar sus aguas en el Océano Pacífico a la altura de Bajos de Chila. Esta cuenca es reconocida como Región Hidrológica Oaxaca-Puerto Angel (INEGI, 1988d).

La otra cuenca, en el extremo N-NE de este sistema, tiene como base los ríos Trapiches y Playas, que al unirse forman el Río Yuguñu. Este río es un tributario importante del Río Atoyac, el cual en esta zona de la sierra tiene una trayectoria hacia el oeste, hasta unirse al Río Verde para desembocar en el Oceáno Pacífico al NO de la bahía de Chacahua (SARH, 1985).

\section{ACTIVIDADES HUMANAS}

En la zona de estudio predominan básicamente dos actividades. Por un lado, la agrícola-ganadera, en la cual destacan el 
cultivo de maíz, además de cafetales en baja proporción en la porción sur. En mucha menor cantidad se producen calabaza, chile y frijol. La agricultura se realiza con el método de roza, tumba y quema, situación que evidentemente pone en peligro a la vegetación del lugar. En cuanto a la ganadería, quizá sea la actividad menos importante, pues sólo un reducido número de familias cuenta con algunas cabezas de ganado vacuno, utilizándolo principalmente para su venta fuera del municipio y sólo en ocasiones como alimento.

La principal actividad y consecuentemente la que más afecta a la zona de estudio, es la maderera ya que desde años atrás las compañías papeleras, como la de Tuxtepec, se han dedicado a la explotación de sus recursos madereros, principalmente el pino, que es el dominante en el área. Observaciones personales ponen de manifiesto que esta actividad es el principal problema para la conservación en el largo plazo de los recursos naturales del lugar, pues en gran parte de la región la explotación forestal se realiza en forma irracional, a lo cual se agrega la inexistencia de un plan de reforestación o la implementación de áreas protegidas, sobre todo en aquellas zonas donde se ha dado una regeneración natural.

\section{MÉTODOS}

Se realizaron 16 salidas a la zona de estudio entre 19881990, lo que significó 40 días efectivos de trabajo de campo. Se procuró hacer la recolección durante todos los meses del año, con el fin de tener cubiertas todas las épocas de floración; el trabajo se llevó a cabo en todos los sitios accesibles y lo más intensiva y exhaustivamente posible. Durante el trabajo de campo se recolectaron 1,900 números. Los tipos de vegetación se determinaron de manera objetiva, tomando los datos sobre la fisonomía de la comunidad en sitios selectos a lo largo y ancho de toda la zona de estudio. Se evaluaron las especies dominantes a simple vista, sin seguir ningún método riguroso.

El material recolectado se procesó y se curó en el Herbario Nacional del Instituto de Biología de la UNAM, donde se encuentra el primer juego; los duplicados del material han sido distribuidos a otros herbarios, como parte del programa de intercambios. Gran parte del material fue revisado por especialistas, lo que da un alto grado de confiabilidad a las determinaciones. En total se estudiaron cerca de 2,250 ejemplares, los cuales documentan la flora de la región.

Los datos de geología, suelos y climas fueron obtenidos principalmente de las cartas temáticas elaboradas para la región por el INEGI. Los límites geográficos se definieron con base en la cartografía disponible sobre topografía (INEGI, 1988a y b) y mediante observaciones realizadas en recorridos de campo.

Para evaluar las afinidades florísticas de la zona de estudio con otras localidades de México, se comparó el listado generado con el de otras 17 regiones del país. Las localidades comparadas fueron arbitrariamente seleccionadas de un conjunto de localidades ya estudiadas florísticamente en el país; ningún criterio específico se utilizó en la selección de las localidades comparadas, excepto que fueron estudiadas recientemente, aumentando así la seguridad de que sus listados florísticos estuvieran taxonómicamente actualizados.

El coeficiente de similitud utilizado para la comparación florística entre las localidades fue el de Simpson (CSS $=\mathrm{c} / \mathrm{a}$, donde $\mathrm{c}$ es el número de taxa compartidos por las dos áreas analizadas y a es el total de taxa encontrados en la muestra más pequeña). Este coeficiente de similitud ha sido utilizado para evaluar niveles de similitud florística 0 faunística entre diferentes áreas; es un coeficiente sencillo de calcular y uno de los más robustos, ya que presenta un comportamiento más consistente de los resultados, especialmente cuando existen notables diferencias en los valores totales de las muestras a comparar (Morafka, 1977; Sánchez y López, 1988).

\section{RESULTADOS}

Los resultados se exponen en tres partes. En la primera se hace una breve descripción de los tipos de vegetación encontrados en la región; en la segunda se incluye un breve análisis de las semejanzas florísticas entre la zona estudiada y otras regiones de México, y en la última parte, incluida a manera de apéndice, se presenta el listado florístico de la zona estudiada.

\section{Tipos de vegetación}

En la zona de estudio es posible distinguir tres tipos de vegetación (sensu Rzedowski, 1978): bosque mesófilo de montaña, bosque tropical caducifolio y bosque de pinoencino. A continuación se describen algunas características de cada uno de ellos.

\section{a) Bosque mesófilo de montaña}

Se localiza en áreas muy restringidas de la zona de estudio. Se desarrolla en cañadas y laderas protegidas, y sus límites altitudinales se sitúan entre los 1,000 y $2,400 \mathrm{msnm}$. En algunas ocasiones puede mezclarse con el bosque de pino-encino. En el área donde se localiza este bosque la época seca es de aproximadamente cuatro meses; sin embargo, durante la mañana y noche se presentan neblinas constantes, con frecuentes lloviznas que mantienen una permanente humedad, sobre todo en el sotobosque. De esta manera se crea un microclima con poca variación, lo cual define la existencia de esta comunidad. En los meses fríos no se presentan heladas, ni siquiera esporádicamente, como sucede en otras zonas con tipos de vegetación similares en el país (Rzedowski, 1978).

El suelo donde se encuentra es nitrosol dístrico, de color amarillo y rojizo, profundo, con una capa gruesa de materia orgánica. El relieve es accidentado, con pendientes suaves y pronunciadas.

Esta comunidad, en la zona de estudio, es la más rica florísticamente, lo que se manifiesta a través de sus estratos. 
El estrato arbóreo, por ejemplo, contiene árboles que varían en altitud entre 7 a $35 \mathrm{~m}$. Helechos arborescentes de la familia Cyatheaceae llegan a alcanzar alturas de alrededor de $10 \mathrm{~m}$. Las especies más representativas son Alnus acuminata, Arbutusxalapensis, Chiranthodendron pentadactylon, Clethra mexicana, Cornus disciflora, Dendropanaxarboreus, Diospyros campechiana, Ficus padifolia, Inga eriocarpa, Ocotea helicterifolia, Oreopanax xalapensis, Ormosia oaxacana, Parathesis brevipes, Picramnia lindeniana, Quercuscandicans, Siparuna andina, Ulmus mexicana, Viburnum elatum y Zinowiewia integerrima.

El estrato arbustivo está bien representado, con especies como Acalypha microcephala, Boehmeria caudata, Bunchosia lanceolata, Chamaecrista glandulosa, Chiococca alba, Cordia spinescens, Croton cortesianus, Deppea hintonii, Desmodium amplifolium, Euphorbia fulgens, Fuchsia arborescens, Gaultheria odorata, Hedyosmum mexicanum, Heliocarpus tomentosus, Hoffmannia cryptoneura, Hyptis oblongifolia, Lobelia aguana, Myrica cerifera, Myriocarpa obovata, Palicourea padifolia, Phenax hirtus, Picramnia lucuples, Piper patzulinum, Psychotria pubescens, Rhus radicans, Rondeletia buddleioides, Rubus trichomallus, Ruellia petiolaris, Salvia adenophora, Solanum acerifolium, Tephrosia lanata, Triumfetta dehiscens, Turnera diffusa, Urera caracasana y Xylosma flexuosum.

Cuando este tipo de vegetación se presenta sin perturbación intensa, las hierbas no son muy abundantes. Sin embargo, en la zona de estudio se da el caso contrario, existiendo una alteración constante. Entre las especies más comunes se pueden citar a Acalypha frederici, Achimenes antirrhina, Apium leptophyllum, Arundinella deppeana, Asclepias circinalis, Barleria micans, Begonia boissieri, Brachistus stramonifolius, Calanthe calanthoides, Calceolaria mexicana, Canna indica, Commelina diffusa, Costus bakeri, Crotalaria bupleurifolia, C. mollicula, Crusea coccinea, Cuphea aequipetala, Cyperus hermaphroditus, Euphorbia oerstediana, Govenia superba, Heliconia collinsiana, Hyptis pectinata, Kyllinga brevifolia, Lamourouxia xalapensis, Lasiacis divaricata, Leucocarpus perfoliatus, Lobeliaaguana, Malaxislepidota, Melinis minutiflora, Oenothera pubescens, Phyllanthus niruri, Ruellia stemonacanthoides, Salvia cinnabarina, Solanum appendiculatum, Tradescantia commelinoides y Viola humilis.

Las epífitas, trepadoras y rupícolas están bien desarrolladas en esta comunidad y existen familias muy ricas y abundantes, como Orchidaceae y Piperaceae. Entre ellas podemoscitar a Achimenesflava, Anthuriumandicola, Blepharodon mucronatum, Catopsis sessiliflora, Cuitlauzina pendula, Dioscorea composita, Encyclia bicamerata, Epidendrum arbuscula, Melothria pendula, Oncidium hastatum, Paullinia pinnata, Pentadenia matudae, Peperomia blanda, Pitcairnia heterophylla, Pleurothallis minutalis, Restrepia muscifera, Rhipsalis baccifera, Tillandsiajuncea, Trichopilia galeottiana y Vitis cinerea. Acompañando a las epífitas, se encuentran en abundancia hepáticas, líquenes, musgos y helechos.

En algunas áreas las hemiparásitas y saprófitas se presentan con relativa frecuencia, probablemente debido a laperturbación. Entre las especies encontradas están Chimaphila maculata, Cladocolea loniceroides, Gymnosiphon suaveolens y Psittacanthus macrantherus.

En los últimos años se ha comenzado a afectar con mayor intensidad este tipo de vegetación. El cambio en el uso del suelo, principalmente para el cultivo de café y en menor porcentaje el de maíz, así como la apertura de nuevas áreas para cultivo por medio del fuego, son las causas principales de la constante desaparición de la vegetación original.

\section{b) Bosque tropical caducifolio}

Este tipo de vegetación forma un manchón aislado al $\mathrm{N}-\mathrm{NE}$ de la zona de estudio, localizándose entre la cota de $\operatorname{los} 1,400$ y $1,500 \mathrm{msnm}$. Se desarrolla en un suelo calizo, pedregoso, de color amarillento y relativamente profundo, derivado de rocas metamórficas, pobre en materia orgánica, sobre pendientes pronunciadas y lomeríos suaves.

Los árboles presentan alturas de 3 a $10 \mathrm{~m}$, dominando las siguientes especies (incluidas algunas riparias): Acacia angustissima, Bursera fagaroides, $B$. galeottiana, $B$. schlechtendalii, Colubrina ehrenbergii, Litsea glaucescens, Lysiloma divaricata, Pistacia mexicana, Psidium guajava, Recchia connaroides, Salix bonplandiana, Sapindus saponaria, Senna mollissima, Styrax polyneurus y Thevetia ovata.

Los arbustos tienen una altura que oscila entre 1 y 4 m y las especies que sobresalen son Amelanchier denticulata, Ayenia manzanilloa, Calliandra eriophylla, Cestrum glanduliferum, Croton cortesianus, Dodonaea viscosa, Euphorbia pulcherrima, Galphimia glauca, Heliotropium ternatum, Lantana hispida, Malpighia mexicana, Solanum chrysotrichum, Tecoma stans, Turnera diffusa y Wigandia urens.

Probablemente, debido a la perturbación existente, la riqueza en elementos herbáceos no es alta. Sin embargo, las poblaciones de algunas especies llegan a ser grandes, sobre todo de los miembros de las familias Asteraceae y Poaceae. Entre las hierbas más comunes están Brickellia glandulosa, Capsicum rhomboideum, Coursetia caribea, Cyrtopodium punctatum, Dahlia coccinea, Fleischmanniopsis leucocephala, Gamochaeta americana, Haplophyton cinereum, Lasiacis nigra, Lithospermum calcicola, Melinis minutiflora, Panicum pulchellum, Peperomia peltata, Physalis sancti-josephi y Polanisia uniglandulosa.

Las epífitas son muy escasas, dominando prácticamente el género Tillandsia. En la misma situación están las trepadoras, donde encontramos a Dioscorea convolvulacea, D. floribunda, D. remotiflora, Passiflora sp., Phaseolus lunatus, Thenardia sp. y Valeriana scandens.

Las cactáceas son un grupo importante en este tipo de vegetación; destacan por ejemplo Mammillaria haageana, M. karwinskiana, Neobuxbaumia mezcalensis, Nopalxochia ackermanii y Stenocereus pruinosus. Por otra parte, en 
barrancas con corriente de agua o de mayor humedad a lo largo del año, se encuentran especies propias de comunidades cálido-húmedas, como es el caso de Piper aduncum, $P$. amalago y P. scabrum.

c) Bosque de pino-encino

Esta formación domina en el área de èstudio. En el NNO de la zona, donde están los picos más altos, como el cerro El Encanto (2,700 m), existe una mezcla con elementos mesófilos, como Ardisia sp., Chiranthodendron pentadactylon, Clusia sp., Daphnopsis nevlingii y Quercus sp., sin ser dominantes ninguno de ellos.

El bosque se desarrolla desde los 1,000 hasta los 2,700 msnm, sobre un suelo denominado nitrosol dístrico ácido y en un clima semicálido subhúmedo. Queda ubicado dentro de los límites climáticos, edáficos y altitudinales que apunta Rzedowski (1978) para este tipo de vegetación.Fisonómicamente presenta alturas que van de los 5 a $\operatorname{los} 20 \mathrm{~m}$.

Los árboles miden entre 10 y $20 \mathrm{~m}$ dominando especies como Alnus acuminata, Pinus maximinoi, P. michoacana, P. oaxacana, $P$. oocarpa, $P$. patula subsp. recunumanii, Quercus elliptica, Q. glaucoides, Q. laurina, Q. magnoliifolia, $Q$. scytophylla y $Q$. urbanii.

Los arbustos presentan alturas de 1 a $4 \mathrm{~m}$ en promedio. Entre las especies más comunes están Acalyphafrederici, Agarista mexicana, Arctostaphylos pyrifolia, Bejaria mexicana, Boehmeria nivea, Calliandra hirsuta, Cestrum glanduliferum, Clidemia matudae, Conostegia xalapensis, Croton cortesianus, Desmodium helleri, Fuchsia arborescens, Iresine celosia, Leandra subseriata, Marina scopa, Miconia glaberrima, Mimosa albida, Moussonia deppeana, Phenax hirtus, Piperaduncum, Rhus schiedeana, Rubus trichomalus, Salvia adenophora, Senna mollissima, Solanum acerifolium, Tephrosia lanata, Triumfetta grandiflora, Vaccinium confertum y Xylosma celastrinum.

Las hierbas llegan a alcanzar alturas hasta de $1.5 \mathrm{~m}$. Entre las especies más frecuentes se pueden citar a Acalypha microcephala, Arenaria alsinoides, Arundinella deppeana, Apium leptophyllum, Begonia boissieri, B. fusca, Castilleja lithospermoides, Clematis dioica, Commelina diffusa, Crotalariaacapulcensis, Crusea coccinea, Cuphea aequipetala, Cyperus hermafroditus, Dalea leucostachys, Diastatea micrantha, Dorstenia contrajerba, Drymaria gracilis, Justicia caudata, Lamourouxia multifida, Lantana hispida, Lasiacis divaricata, Lobelia laxiflora, Maianthemum paniculatum, Muhlenbergia robusta, Oplismenus hirtellus, Phaseolus chiapasanus, Phyllanthus niruri, Physalis caudella, Pinguicula heterophylla, Russelia coccinea, Satureja macrostema, Sida rhombifolia, Tibouchina scabriuscula, Tradescantia zanonia y Valeriana sorbifolia.

Las epífitas dominantes pertenecen a las familias Bromeliaceae, Orchidaceae y Piperaceae, aunque en general no existe una gran riqueza de ellas. Las especies encontradas con mayor frecuencia son Encyclia bicamerata, Epidendrum ramosum, Oncidium hastatum, Peperomia blanda, P. galioides, P. obtusifolia, P. quadrifolia, Tillandsia butzii y T. usneoides. Acompañando a estas especies, se encuentran también algunas hepáticas, musgos y helechos que incluso llegan a ser más abundantes. Las trepadoras son escasas, y las más comunes son Bomarea hirtella, Dioscorea composita, Passiflora cookii, Valeriana scandens y Vitis tiliifolia.

\section{Recuento florístico}

De la determinación del material colectado, se reconocieron 680 especies, repartidas en 370 géneros y 123 familias (Cuadro 1). Las familias mejor representadas en la región son Orchidaceae, Asteraceae, Piperaceae, Fabaceae y Rubiaceae, que juntas comprenden el $30.1 \%$ de la flora. Las familias incluidas en el Cuadro 1 aportan el $52.6 \%$ del total de especies. Por su parte, los géneros con el mayor número de especies en la zona son Piper (21), Peperomia (17), Quercus (16), Salvia (15), Pinus (9), Encyclia (8), Begonia (7), Crotalaria (7), Cuphea (7), Dioscorea (7) e Ipomoea (7). En el apéndice de este trabajo se presenta la lista florística, ordenada según el sistema de clasificación de Cronquist (1981).

CuAdro 1. Total de familias, géneros y especies presentes en la porción central del Municipio de San Jerónimo Coatlán, Oaxaca y familias con mayor número de especies.

\begin{tabular}{lcc}
\hline Familias & Géneros & Especies \\
\hline Orchidaceae & 34 & 53 \\
Asteraceae & 36 & 45 \\
Piperaceae & 2 & 38 \\
Fabaceae & 19 & 37 \\
Rubiaceae & 19 & 32 \\
Lamiaceae & 5 & 20 \\
Mimosaceae & 7 & 18 \\
Fagaceae & 1 & 16 \\
Euphorbiaceae & 6 & 15 \\
Melastomataceae & 9 & 15 \\
Poaceae & 9 & 14 \\
Solanaceae & 6 & 13 \\
Myrsinaceae & 4 & 11 \\
Pinaceae & 2 & 11 \\
Cactaceae & 8 & 10 \\
Scrophulariaceae & 8 & 10 \\
Otras & 195 & 322 \\
& & 680 \\
TOTAL, 123 & 370 &
\end{tabular}

En el Cuadro 2 se muestra la distribución de las especies, de acuerdo con su forma de crecimiento, entre los tres diferentes tipos de vegetación de la zona. El $81.1 \%$ se encuentra restringido a un solo tipo de vegetación, siendo el bosque mesófilo de montaña la comunidad que cuenta con 
CUADRO 2. Distribución por formas de crecimiento y por tipos de vegetación de las especies de fanerógamas de la porción central del Municipio de San Jerónimo Coatlán, Oaxaca.

Tipo de

vegetación ${ }^{1}$

Hierbas $^{2}$

Arbustos

Árboles

TOTAL

$\begin{array}{lcccc}\text { BMM } & 197 & 95 & 61 & 353 \\ \text { BPE } & 63 & 32 & 17 & 112 \\ \text { BTC } & 47 & 22 & 18 & 87 \\ \text { BMM y BPE } & 65 & 37 & 14 & 116 \\ \text { BMM y BTC } & 3 & 3 & 1 & 7 \\ \text { BMM, BPE y BTC } & 1 & 1 & 1 & 3 \\ \text { BPE y BTC } & 1 & 0 & 1 & 2 \\ \text { TOTAL } & \overline{377} & \overline{190} & \overline{113} & \overline{680}\end{array}$

'BMM=bosque mesófilo de montaña, BPE = bosque de pino-encino, BTC=bosque tropical caducifolio.

${ }^{2}$ Como hierbas se incluyen las epífitas, las plantas trepadoras y los sufrútices.

CUADRo 3. Provincia florística a la que pertenece, superficie que ocupa, tipos de vegetación que contiene y número de géneros y especies de algunas regiones de México seleccionadas para realizar comparaciones florísticas.

\begin{tabular}{llllll}
\hline Localidad & $\begin{array}{l}\text { Provincia } \\
\text { Florística }^{1}\end{array}$ & $\begin{array}{l}\text { Superficie } \\
\text { (Hectáreas) }\end{array}$ & $\begin{array}{l}\text { Tipo de } \\
\text { Vegetación }\end{array}$ & Géneros & Especies \\
\hline San Jerónimo Coatlán & SM & 17,300 & BMM, BPE, BTC & 370 & 680 \\
Sierra de la Laguna, BCS & PBC & 1,000 & BPE, BC & 170 & 253 \\
Sierra de San Carlos, Tams. & PCNO, SMOR & 14,226 & BJ, BPE & 298 & 391 \\
El Cielo, Tams. & SMOR & 30,000 & BMM & 147 & 165 \\
La Michilía, Dgo. & SMOC & 70,000 & BPE & 345 & 682 \\
Bahía de Chamela, Jal. & CPA & 7,000 & BTC, VA & 535 & 699 \\
Huehuetoca, Méx. & SM & 1,000 & MXE, PA, VA & 316 & 556 \\
Zoquiapan, Méx. & SM & 2,845 & BC & 180 & 327 \\
Pedregal de San Angel, D. F. & SM & 124.5 & MXE & 242 & 423 \\
Ocuilan, Méx.-Mor. & SM & 15,000 & BMM & 120 & 160 \\
La Vainilla, Gro. & CPA & 344 & BTC, BTSC & 164 & 414 \\
Teocelo, Ver. & SM & 22,000 & BMM & 422 & 275 \\
Xalapa, Ver. & CGM & 31,900 & BMM, BTC, BTSC, VA & 731 \\
Sierra de Chiconquiaco, Ver. & CGM & 5,250 & BMM, BC, BTP & 942 \\
Pico de Orizaba, Ver. & SM & 16,400 & BMM, BPE, PA & 565 & 537 \\
Sierra de San Felipe, Oax. & SM & 12,000 & BPE & 328 & 371 \\
Cerro Guiengola, Oax. & CPA & 4,530 & BC, BTC & 271 & 454 \\
Sian Ka'an & PY & 528,000 & BTSC, BTSP, VDC, VA & 471 & 856
\end{tabular}

' Provincias florísticas (Rzedowski, 1978): CGM= Costa del Golfo de México, CPA= Costa del Pacífico, PBC= Península de Baja California, $\mathrm{PCNO}=$ Planicie Costera del Noroeste, $\mathrm{PY}=$ Península de Yucatán, $\mathrm{SM}=$ Serranías Meridionales, SMOC= Sierra Madre Occidental, SMOR $=$ Sierra Madre Oriental

${ }^{2}$ Tipos de Vegetación: $\mathrm{BC}=$ bosque de coníferas, $\mathrm{BJ}=$ bosque de Juniperus, $\mathrm{BMM}=$ bosque mesófilo de montaña, $\mathrm{BPE}=$ bosque de pinoencino, $\mathrm{BTP}=$ bosque tropical perennifolio, $\mathrm{BTSC}=$ bosque tropical subcaducifolio, $\mathrm{BTSP}=$ bosque tropical subperennifolio, $\mathrm{MXE}=$ matorral xerófilo, $\mathrm{PA}=$ páramo de altura, $\mathrm{BTC}=$ bosque tropical caducifolio, $\mathrm{BTSC}=$ bosque tropical subcaducifolio, $\mathrm{VA}=$ vegetación acuática, $\mathrm{VDC}=$ vegetación de dunas costeras. 
el mayor número. Por otra parte, las especies que se encuentran en dos o más comunidades representan el 18.9\%; ellas son compartidas en mayor escala entre el bosque mesófilo de montaña y el bosque de pino-encino.

\section{Afinidades floristicas}

En el Cuadro 3 se muestra la riqueza tanto genérica como específica de la región estudiada y de otras 17 regiones de México, los tipos de vegetación que contienen, la superficie que ocupan, así como la provincia florística a la que pertenecen (Rzedowski, 1978). Ocho de ellas se ubican en la provincia de las Serranías Meridionales: San Jerónimo Coatlán, Ocuilan (Luna et al., 1989b), Pedregal de San Angel (Valiente-Banuet y de Luna, 1990), Pico de Orizaba (Martínez, 1988), Teocelo (Luna et al., 1989a), Huehuetoca (Romero y Rojas, 1991), Zoquiapan (Vega, 1982) y Sierra de San Felipe (Saynes, 1989). Tres localidades se ubican en la Costa Pacífica: Chamela (Lott, 1993), Cerro Guiengola (Torres, 1989) y La Vainilla (Gallardo, 1992). Por otra parte, la Sierra de San Carlos (Briones, 1991) y El Cielo (Puig y Bracho, 1987) son localidades de la Sierra Madre Oriental; Chiconquiaco (Gutiérrez, 1993) y Xalapa (Zola, 1987), de la Planicie Costera del Golfo de México; la Michilía (González-Elizondo et al., 1993), de la Sierra Madre Occidental; la Sierra de la Laguna (León de la Luz y Domínguez-Cadena, 1989), de la península de Baja California y Sian Ka'an (Durán y Olmsted, 1987) de la península de Yucatán.
En el Cuadro 3 se observa que entre más tipos de vegetación incluya una región, más riqueza florística contiene. Tal correlación puede deberse o estar relacionada con la diversidad de gradientes altitudinales, orográficos, edáficos y climáticos, lo cual permite la existencia de diversos ambientes y el establecimiento de diferentes comunidades vegetales, aumentando así la riqueza florística del lugar. Por otro lado, las regiones con un tipo de vegetación presentan menor número de taxa; esto no quiere decir que su riqueza y diversidad es menor, sino que el muestreo realizado sólo en un tipo de vegetación reduce la diversidad producida por la heterogeneidad ambiental. Lo anterior puede verse en localidades como Teocelo, El Cielo y Ocuilan; una notable excepción es el Pedregal de San Ángel que, aunque cuenta con un solo tipo de vegetación (matorral xerófilo), en una superficie de apenas 124.5 hectáreas, contiene un elevado número de taxa.

En el Cuadro 4 se presenta el porcentaje de similitud, utilizando el coeficiente de similitud de Simpson que tiene la región estudiada con cada una de las localidades del Cuadro 3. Los valores se indican tanto al nivel genérico como específico.

En nivel genérico los resultados muestran que la región estudiada tiene una alta similitud florística con algunas regiones comparadas. Tal es el caso de El Cielo, Ocuilan, Teocelo y Chiconquiaco. En esas áreas el uso del coeficiente produjo valores mayores al $50 \%$ de similitud. Es interesante observar que no todas ellas pertenecen a la misma provincia florística; tienen en común que el bosque mesófilo de mon-

CuAdro 4. Número de taxa compartidos y coeficientes de similitud (Simpson) entre San Jerónimo Coatlán y otras regiones de México.

$\begin{array}{ll}\text { Localidad } & \begin{array}{l}\text { Géneros } \\ \text { compartidos }\end{array}\end{array} \quad \begin{aligned} & \text { Coeficiente } \\ & \text { (géneros) }\end{aligned}$

(\%)

$\begin{array}{ll}\text { Especies } & \text { Coeficiente } \\ \text { compartidas } & \text { (especies) }\end{array}$

$(\%)$

\begin{tabular}{|c|c|c|c|c|}
\hline San Jerónimo Coatlán & 370 & 100 & 680 & 100 \\
\hline Sierra de la Laguna & 69 & 40.6 & 12 & 4.7 \\
\hline Sierra de San Carlos & 106 & 35.6 & 30 & 7.7 \\
\hline El Cielo & 81 & 55.1 & 25 & 15.2 \\
\hline La Michilía & 106 & 30.7 & 38 & 5.6 \\
\hline Chamela & 140 & 37.8 & 56 & 8.2 \\
\hline Huehuetoca & 94 & 29.8 & 34 & 6.1 \\
\hline Zoquiapan & 52 & 28.9 & 20 & 6.1 \\
\hline Pedregal de San Angel & 98 & 40.5 & 39 & 9.2 \\
\hline Ocuilan & 83 & 69.2 & 36 & 22.5 \\
\hline La Vainilla & 111 & 37.8 & 25 & 6.0 \\
\hline Teocelo & 108 & 64.3 & 48 & 17.5 \\
\hline Xalapa & 176 & 47.6 & 99 & 14.6 \\
\hline Sierra de Chiconquiaco & 204 & 55.1 & 139 & 20.4 \\
\hline Pico de Orizaba & 156 & 47.6 & 83 & 15.5 \\
\hline Sierra de San Felipe & 109 & 40.2 & 86 & 23.2 \\
\hline Cerro Guiengola & 103 & 37.1 & 25 & 5.5 \\
\hline Sian Ka'an & 129 & 34.9 & 60 & 8.8 \\
\hline
\end{tabular}


Cuadro 5. Número de especies compartidas por tipo de vegetación entre San Jerónimo Coatlán, Oaxaca y otras regiones de México, total de especies presentes en la localidad y porcentaje de similitud (utilizando el coeficiente de Simpson).

$\begin{array}{lrrrrr}\text { Localidad } & \text { BMM } & \text { BPE } & \text { BTC } & \text { TOTAL } & \text { Similitud } \\ & & & & & \\ \text { San Jerónimo Coatlán } & 353 & 112 & 87 & 551 & 100 \\ \text { Sierra de la Laguna } & & 5 & & 253 & 2.0 \\ \text { Sierra de San Carlos } & & 7 & & 391 & 2.0 \\ \text { El Cielo } & 18 & & & 65 & 27.7 \\ \text { La Michilía } & & 20 & & 682 & 5.7 \\ \text { Chamela } & & & 18 & 699 & 3.1 \\ \text { Zoquiapan } & 24 & & & 327 & 15.0 \\ \text { Ocuilan } & 50 & & & 160 & 18.2 \\ \text { Teocelo } & & 83 & & 275 & 23.5 \\ \text { San Felipe } & & & 19 & 454 & 5.4 \\ \text { Guiengola } & & & & & \end{array}$

taña es el tipo de vegetación dominante, o al menos el tipo de vegetación que alberga la mayor riqueza florística en la región.

Es notable observar que en el nivel específico los valores de similitud son extremadamente bajos, sin importar lo cercano o lejano de las regiones con San Jerónimo Coatlán. Las especies compartidas entre las regiones analizadas son muy pocas y aun aquellas que presentaban un alto coeficiente de similitud con respecto al número de géneros compartidos, muestran muy poca correspondencia en el nivel de especies.

Por el número de especies compartidas entre San Jerónimo Coatlán y las demás áreas, ninguna formaría parte de la misma región fitogeográfica. Las regiones con las que presenta los más altos coeficientes son la Sierra de San Felipe, Ocuilan y la Sierra de Chiconquiaco. Aunque San Felipe no presentó un valor de similitud alto con respecto a los géneros compartidos, es la región con mayor coeficiente cuando se evalúan las especies.

En el Cuadro 5 se presentan los porcentajes de similitud entre San Jerónimo Coatlán y otras regiones, cuando se comparan los elementos exclusivos de un solo tipo de vegetación. De esta manera, se determinó el número de especies exclusivas del bosque mesófilo de montaña, del bosque de pino-encino o del bosque tropical caducifolio en la región de estudio, que además se comparten con tipos de vegetación equivalentes en otras regiones evaluadas. También en el Cuadro 5 se destaca el pequeño número de especies compartidas entre las zonas. Es difícil determinar por qué el número de especies compartidas es tan bajo con base en apreciaciones como las aquí realizadas. Sin embargo, es importante mencionar que biotas que comúnmente se reconocen como un mismo tipo de vegetación, de acuerdo con observaciones o evaluaciones fisonómicas, están constituidas por géneros o especies distintas. De esta manera, hablar por ejemplo del bosque mesófilo de San Jerónimo Coatlán, El Cielo, Ocuilan o Teocelo, implica definir una comunidad con ciertos atri- butos fisonómicos y estructurales, pero generalmente con una composición florística muy diferente.

\section{DISCUSIÓN}

El trabajo de inventario realizado en esta pequeña porción de la Sierra Madre del Sur revela la notable riqueza florística que seguramente contiene toda esta provincia fisiográfica de México. Por otra parte, las comparaciones de esta flora con otras regiones del país, manifiestan lo diferente que son cada una de ellas en cuanto a su composición florística, aunque contengan un mismo tipo de vegetación.

Estudios en pequeñas regiones, como la aquí estudia$\mathrm{da}$, son fundamentales para entender lo compleja y vasta que es la flora mexicana. Igualmente presentan elementos para comprender por qué nuestro país contiene tan alto número de especies endémicas, muchas de ellas restringidas a pequeñas regiones (microendemismos). Por ejemplo, para la zona de San Jerónimo Coatlán cerca del $20 \%$ de su flora es endémica al territorio nacional, alrededor de 41 especies son endémicas al estado de Oaxaca, 12 de ellas hasta la fecha sólo se conocen del Distrito de Miahuatlán y tres especies son únicamente conocidas del municipio (Deppea miahuatlana, Lepanthes camposii y Salvia nicolsoniana).

Sólo con más estudios locales se podrán hacer mejores generalizaciones acerca de los patrones biogeográficos de las especies y biotas en general. Como Hengeveld (1990) argumenta, las respuestas al porqué una o más especies se presentan en una región y por qué su área de distribución tiene ciertas dimensiones, sólo podrán buscarse estudiando las condiciones locales dentro de esta área, así como los factores históricos que determinaron sus condiciones actuales. También es recomendable que futuros estudios determinen los valores de semejanza florística entre la zona estudiada y otras regiones más o menos semejantes y se defina claramente el nivel taxonómico en el que se está comparando. Como se ha demostrado, el coeficiente de similitud 
revela datos muy interesantes de relación de comunidades con respecto al nivel de jerarquía taxonómica que se esté usando.

\section{AGRADECIMIENTOS}

La Dra. Patricia Dávila y los M. en C. Hilda Flores, Guillermo Ibarra, Jaime Jiménez, y Helga Ochoterena, revisaron una primera versión de este trabajo; se agradece los valiosos comentarios que hicieron para mejorarlo. El material fue revisado y determinado por numerosos especialistas; especialmente agradecemos la colaboración de Frank Almeda (Melastomataceae), Thomas F. Daniel (Acanthaceae), Nelly Diego (Cyperaceae), Paul Fryxell (Malvaceae), Abisaí García (Agavaceae, Amaryllidaceae y Liliaceae), Shirley A. Graham (Lythraceae), Celso Gutiérrez(Heliconiaceae), Héctor Hernández (Mimosaceae), David Hunt (Commelinaceae), Guillermo Ibarra(Moraceae), Verónica Juárez(Asclepiadaceae), Myron Kimnach (Crassulaceae), Leslie R. Landrum (Myrtaceae), Rafael Lira (Cucurbitaceae), David Lorence (Rubiaceae), Esteban Martínez (Burmanniaceae), Isidro Méndez (Scrophulariaceae), Michael Nee (Solanaceae), T. P. Ramammoorthy (Lamiaceae), Angelica RamÍrez (Gesneriaceae), Lourdes Rico (Mimosaceae), Jerzy Rzedowski (Burseraceae), Gerardo Salazar (Orchidaceae), Jorge Sánchez-Ken (Poaceae), Carmen Soto (Fabaceae), Mario Sousa (Fabaceae), W. D. Stevens (Asclepiadaceae), Brian Styles (Pinaceae), Oswaldo Téllez (Dioscoreaceae, Fabaceae), Leticia Torres (Apiaceae), John Utley (Bromeliaceae), K. B. Utley (Begoniaceae), Grady L. Webster (Euphorbiaceae), Henk van derWerff (Lauraceae) y Sergio Zamudio (Lentibulariaceae).

En sus últimas etapas, este trabajo contó con apoyo económico de DGAPA-UNAM (Proyecto IN204292) y de CONACYT (Proyecto 400355-5-3012-N).

\section{LITERATURA CITADA}

Briones V OL. 1991. Sobre la flora, vegetación y fitogeografía de la Sierra de San Carlos, Tamaulipas. Acta Botánica Mexicana 16: $15-43$.

Brown JH, Gibson AC. 1983. Biogeography. The C. V. Mosby Co. Saint Louis, Missouri.

Conzatti C. 1918. Exploración botánica por la costa meridional de Oaxaca. Boletín de la Dirección de Estudios Biológicos México 2: 309-325.

Conzatti C. 1922. Una expedición botánica a la costa oaxaqueña del suroeste. Imprenta del Gobierno del Estado de Oaxaca, Oaxaca.

Conzatti C. 1929. Las regiones botánico-geográficas del estado de Oaxaca. Proceedings of the International Congress of Plant Sciences (Ithaca) 1: 525-539.

Cronquist A. 1981. An integrated system of classification of flowering plants. Columbia University. Press, New York.

Dávila AP, Germán R MT. 1991. Herbario Nacional de México. Colecciones Biológicas Nacionales del Instituto de Biología, México, Instituto de Biología, UNAM, México.

Durán GR, Olmsted I. 1987. Listado florístico de la Reserva de Sian Ka'an. Amigos de Sian Ka'an. Puerto Morelos, Quintana Roo.

Gallardo HC. 1992. Estudio de la flora y vegetación del parque ecológico «La Vainilla», Zihuatanejo, Guerrero. Tesis de Licenciatura, Facultad de Ciencias, UNAM, México.
García A, Torres R. (En prensa). Estado actual del conocimiento de la flora de Oaxaca. Serie Flora de Oaxaca, Instituto de Biología, UNAM, México.

García E. 1981. Modificaciones al sistema de clasificación climático de Köppen. Instituto de Geografía, UNAM, México.

González-Elizondo S, González-Elizondo M, Cortés-Ortiz A. 1993. Vegetación de la reserva de la biósfera «La Michilía», Durango, México. Acta Botánica Mexicana 22: 1-104.

Gutiérrez BC. 1993. Listado florístico de la Sierra de Chiconquiaco, Ver. Textos Universitarios. Universidad Veracruzana, Xalapa.

Hengeveld R. 1990. Dynamic biogeography. Cambridge Studies in Ecology. Cambridge University Press, Cambridge.

INEGI. 1985. Carta de efectos climáticos. 1: 250,000. Zaachila. E1412. SPP, México.

INEGI. 1988a. Carta topográfica. 1: 50,000. San Baltazar Loxicha. E14D87. SPP, México.

INEGI. 1988b. Carta topográfica. 1: 50,00(). Santa Catarina Juquila. E14D86. SPP, México.

INEGI. 1988c. Carta geológica. 1: 250,000. Zaachila. E14-12. SPP, México.

INEGI. 1988d. Carta hidrológica de aguas superficiales. 1: 250,000. Zaachila. E14-12. SPP, México.

León de la Luz JL, Domínguez-Cadena R. 1989. Flora of the Sierra de la Laguna, Baja California Sur, Mexico. Madroño 36: 6183.

López-Ramos E. 1974. Carta geológica del estado de Oaxaca. 2a. ed. Comité de la Carta Geológica de la República Mexicana. Escala: 1: 500000.

Lorence DH, García MA. 1989. Oaxaca, México. En: Campbell DG, Hammond $\mathrm{HD}$, eds. The world wild life fund strategy for the inventory of tropical forests. New York Botanical Garden, Bronx, New York.

Lott EJ. 1993. Annotated checklist of the vascular flora of the Chamela Bay region, Jalisco, Mexico. Occasional Papers of the Californy Academy of Sciences No. 148, San Francisco, California.

Luna I, Almeida L, Villers I, Lorenzo L. 1989a. Reconocimiento florístico y consideraciones fitogeográficas del bosque mesófilo de montaña, Teocelo, Veracruz. Boletín de la Sociedad Botánica de México 48: 35-63.

Luna I, Almeida L, Villers I, Lorenzo L. 1989b. Florística y aspectos fitogeográficos del bosque mesófilo de montaña de las cañadas de Ocuilan, estados de Morelos y México. Anales del Instituto de Biología, UNAM, Serie Botánica 59: 63-87.

Martínez Y P JL. 1988. La vegetación de la zona noreste del Pico de Orizaba, Veracruz. Tesis de Licenciatura, Facultad de Biología, UDICBA, Xalapa.

Morafka DJ. 1977. A biogeographical analyis of the Chihuahuan Desert through its herpetofauna. Biogeographica. Vol. IX.Dr. W. Junk B. V. Publ., La Haya.

Puig H, Bracho R. 1987. El bosque mesófilo de montaña de Tamaulipas. Instituto de Ecología A.C., Xalapa.

Romero RS, Rojas Z EC. 1991. Estudio florístico de la región de Huehuetoca, Estado de México. Acta Botánica Mexicana 14: 33-57.

Rzedowski J. 1978. Vegetación de México. Ed. Limusa, México.

Sánchez O, López G. 1988. A theoretical analysis of some indices of similarity as applied to biogeography. Folia Entomológica Mexicana 75: 119-145.

SARH. 1985. Inventario Forestal del estado de Oaxaca. Secretaría de Agricultura y Recursos Hidráulicos. Publicación Especial, México.

Saynes VA. 1989. Contribución al conocimiento florístico y fitogeográfico de la vertiente sur de la Sierra de San Felipe, Dto. Centro. 
Oaxaca. Tesis de Licenciatura, Escuela Nacional de Estudios Profesionales «Zaragoza», UNAM, México.

Secretaría de Gobernación y Gobierno del Estado de Oaxaca. 1988. Enciclopedia de los Municipios de Oaxaca. Oaxaca.

Torres CL. 1989. Estudio florístico y descripción de la vegetación del Cerro Guiengola en el Istmo de Tehuantepec, Oaxaca. Tesis de Licenciatura, Escuela Nacional de Estudios Profesionales «Iztacala», UNAM, Izatacala.

Valiente-Banuet A, De Luna E. 1990. Una lista florística actualizada para la reserva del Pedregal de San Angel. Acta Botánica Mexicana 9: 13-30.

Vega AR. 1982. Manual de la flora de la estación experimental de enseñanza e investigación y servicios forestales Zoquiapan. Tesis de Maestría en Ciencias, Colegio de Posgraduados, Chapingo.

Zola M. 1987. La vegetación de Xalapa, Veracruz. Instituto Nacional de Investigaciones Sobre Recursos Bióticos, Xalapa.

\section{APÉNDICE. LISTA FLORÍSTICA DEL MUNICIPIO DE SAN JERÓNIMO COATLÁN, OAXACA.}

Abreviaturas: $\mathrm{H}=$ hierbas (incluyendo epífitas, trepadoras y sufrútices), $\mathrm{S}=$ arbustos, $\mathrm{T}=$ árboles, $\mathrm{BMM}=$ bosque mesófilo de montaña, $\mathrm{BPQ}=$ bosque de pino-encino, $\mathrm{BTC}=$ bosque tropical caducifolio.

\section{DIVISIÓN CONIFEROPHYTA}

\section{Familia Cycadaceae}

Dioon purpusii Rose (T; BTC)

Familia Pinaceae

Abies hickeli Flous \& Gausen (T; BPQ)

Pinus chiapensis (MartÍnez) Andresen (T; BPQ)

Pinus devoniana Lindl. (T; BPQ)

Pinus lawsonii Roesl ex Gordon (T; BPQ)

Pinus leiophylla Schldl. \& Cham. var. leiophylla (T; BPQ)

Pinus maximinoi $\mathrm{H}$.E. Moore (T; BPQ)

Pinus oaxacana Mirov (T; BPQ)

Pinus oocarpa Schiede ex Schldl. var. oocarpa (T; BPQ)

Pinus patula Schldl. \& Cham. subsp. recunumani (Eguiluz \& Perry) Styles (T; BPQ)

Pinus pseudostrobus Lindl. var. pseudostrobus (T;BPQ)

Familia Taxodiaceae

Taxodium mucronatum Tenore (T; BTC)

\section{DIVISIÓN MAGNOLIOPHYTA CLASE LILIOPSIDA}

\section{Familia Agavaceae}

Agave americana L. var. oaxacensis Gentry (H; BTC)

Agave atrovirens Karw. ex Salm-Dyck (H; BTC)

Agave marmorata Roezl (H; BTC)

Agave potatorum Zucc. (H; BTC)

Agave seemanniana Jacobi (H; BTC)

Furcraea guerrerensis Matuda (H; BMM, BPQ)

Furcraea longaeva Karw. \& Zucc. (H; BMM)

Familia Alstroemeriaceae

Bomarea hirtella (Kunth) Herb. (H; BMM, BPQ)

Familia Amaryllidaceae

Sprekelia formosissima (L.) Herb. (H; BPQ, BTC)

Zephyranthes lindleyana Herb. (H; BTC)

Familia Anthericaceae

Echeandia mexicana Cruden (H; BMM, BPQ) 


\section{Familia Araceae}

Anthurium andicola Liebm. (H; BMM)

Anthurium scandens (Aubl.) Engler (V; BMM)

Anthurium schlechtendalii Kunth subsp. jimenezii (Matuda) Croat (H; BMM)

\section{Familia Arecaceae}

Chamaedorea elegans Mart. (H; BMM)

Chamaedorea humilis Mart. (H; BMM)

Chamaedorea karwinskiana Wendl. (H; BMM)

Chamaedorea nubium Standl. \& Steyerm. (H; BMM)

Chamaedorea sartorii Liebm. (H; BMM)

Chamaedorea schiedeana Mart. (H; BMM)

Chrysophila argentea Bartlett (SS; BMM)

\section{Familia Bromeliaceae}

Catopsis sessiliflora (Ruiz \& Pav.) Mez (E; BMM, BPQ)

Pitcairnia heterophylla (Lindl.) Beer (H; BMM)

Tillandsia butzii $\mathrm{Mez}$ (E; BPQ)

Tillandsia ghiesbreghtii Baker (E; BPQ)

Tillandsia guatemalensis L.B. Sm. (E; BPQ)

Tillandsia juncea (Ruiz \& Pav.) Poir. ex Lam. (E; BMM, BPQ)

Tillandsia usneoides L. (E; BMM)

Vriesia werckleana Mez (E; BMM)

\section{Familia Burmanniaceae}

Gymnosiphon suaveolens (Karsten) Urban (H; BMM)

Familia Cannaceae

Canna indica L. (H; BMM)

Familia Commelinaceae

Commelina coelestis Willd. (H; BMM)

Commelina diffusa Burmann f. (H; BMM)

Commelina tuberosa $\mathrm{L}$. (H; BMM)

Gibasis pellucida (M. Martens \& Galeotti) D.R. Hunt (H; BMM)

Thyrsanthemum bracteosum D.R. Hunt (H; BPQ)

Thyrsanthemum floribundum (M. Martens \& Galeotti) Pichon (H; BMM)

Tradescantia commelinoides Roem. \& Schult. (H; BMM, BPQ)

Tradescantia zanonia (L.) Sw. (H; BMM, BPQ)

\section{Familia Convallariaceae}

Maianthemum paniculatum (M. Martens \& Galeotti) LaFrankie (H; BMM, BPQ)

Maianthemum scilloideum (M. Martens \& Galeotti) LaFrankie (H; BMM, BPQ)

\section{Familia Costaceae}

Costus bakeri Schumann (H; BMM)

Costus pictus D. Don (H; BMM)

Familia Cyperaceae

Cyperus hermaphroditus (Jacq.) Standl. (H; BMM)

Cyperus surinamensis Rottb. (H; BMM)

Eleocharis acicularis (L.) Roem. \& Schult. (H; BPQ)

Kyllinga brevifolia Rottb. (H; BMM)

Kyllinga pumila Michx. (H; BMM)

\section{Familia Dioscoreaceae}

Dioscorea compositae Hemsl. (H,V; BMM)

Dioscorea convolvulacea Cham. \& Schldl. (V; BPQ)

Dioscorea dugesii B.L. Rob. (V; BMM, BPQ)

Dioscorea floribunda M. Martens \& Galeotti (V; BMM, BPQ) 
Dioscorea nelsonii Uline ex Kunth (V; BMM)

Dioscorea remotiflora Uline ex Kunth (V; BMM)

Dioscorea ulinei Greene (V; BPQ)

Familia Heliconiaceae

Heliconia collinsiana Griggs var. collinsiana (H; BMM)

Heliconia schiedeana Klotzsch (H; BMM)

Familia Iridaceae

Orthrosanthus monadelphus Ravenna (H; BTC)

Familia Juncaceae

Juncus ebracteatus Liebm. (H; BPQ)

Familia Melanthaceae

Schoenocaulon officinale (Schldl. \& Cham.) A. Gray ex Benth. (H; BPQ)

Familia Nolinaceae

Nolina longifolia (Schultes) Hemsl. (T; BPQ)

Familia Orchidaceae

Barkeria vanneriana Reichb. f. (H; BPQ)

Bletia gracilis Lodd. (H; BMM)

Bletia reflexa Lindl. (H; BMM)

Bulbophyllum cirrhosum L.O. Wms. (E; BPQ)

Calanthe calanthoides (A. Rich. \& Galeotti) Hamer \& Garay (H; BMM)

Campylocentrum schiedei (Reichb. f.) Benth. ex Hemsl. (E; BMM)

Cranichis apiculata Lindl. (H; BMM)

Cuitlauzina pendula Llave (E; BMM)

Cyclopogon elatus (Sw.) Schldl. (H; BMM)

Cypripedium irapeanum Llave \& Lex. subsp. molle (Lindl.) Cribb \& Soto (H; BPQ)

Cyrtopodium punctatum (L.) Lindl. (H; BTC)

Dichaea squarrosa Lindl. (E; BMM)

Encyclia bicamerata (Reichb. f.) Dressler \& Pollard (E; BPQ)

Encyclia chondylobulbon (A. Rich. \& Galeotti) Dressler \& Pollard (E; BMM)

Encyclia magnispatha (A.H.S.) Dressler (E; BPQ)

Encyclia michuacana (Llave) Schltr. (H; BMM)

Encyclia obpiribulbon Hagsater (H; BTC)

Encyclia ochracea (Lindl.) Dressler (E; BPQ)

Encyclia pseudopygmaea (A. Finet) Dressler \& Pollard (E; BMM)

Encyclia varicosa (Lindl.) Schltr. (H; BPQ)

Epidendrum camposii Hagsater (H; BMM)

Epidendrum gasteriferum Scheeren (E; BPQ)

Epidendrum nitens Reichb. f. (E; BPQ)

Epidendrum ramosum Jacq. (E; BMM)

Epidendrum scriptum A. Rich. \& Galeotti (E; BMM)

Govenia mutica (Lindl.) Reichb. f. (H; BMM)

Govenia superba (Llave \& Lex.) Lindl. ex Loddiges (H; BMM)

Hexisea imbricata (Lindl.) Reichb. f. (E; BMM)

Jacquiniella teretifolia (Sw.) Britt. \& Wilson (E; BMM)

Lycaste crinita Lindl. (E; BMM)

Macroclinium lexarzanum (Hagsater \& G. Tamayo) Dodson (E; BMM)

Malaxis histionantha (Link, Klotsch \& Otto) Garay \& Dunsterv. (H; BMM)

Malaxis lepidota (Finet) Ames (H; BMM)

Maxillaria ringens Reichb. f. (E; BMM)

Maxillaria variabilis Batem. ex Lindl. (E; BMM)

Oncidium hastatum (Batem.) Lindl. (E; BMM)

Oncidium hyalinobulbon Llave (E; BPQ)

Oncidium oblongatum Lindl. (E; BMM) 
Oncidium reichenheimii (Linden \& Reichb. f.) Garay \& Stacy (E; BMM, BPQ)

Pleurothallis minutalis Lindl. (E; BMM)

Pleurothallis tubata (Lodd.) Steudel (E; BMM)

Ponthieva ephippium Reichb. f. (H; BMM)

Restrepia muscifera Reichb. f. ex Lindl. (E; BMM)

Restrepiella ophiocephala (Lind1.) Garay \& Dunsterv. (E; BMM)

Rossioglossum insleayi (Barker ex Lindl.) Garay \& Kennedy (E; BMM, BPQ)

Sarcoglottis rosulata (Lindl.) P.N. Donn (H; BTC)

Sobralia macrantha Lindl. (H; BMM)

Stanhopea martiana Bateman ex Lindl. (H; BMM)

Stelis aprica Lindl. (E; BMM)

Stenorrhynchos aurantiacus (Llave) Lindl. (H; BPQ)

Trichocentrum hoegei Reichb. f. (E; BMM)

Trichopilia galeottiana A. Rich. (E; BMM)

Trichosalpinx blaisdellii (S. Watson) Luer (E; BMM)

\section{Familia Poaceae}

Arundinella deppeana Nees (H; BMM)

Lasiacis divaricata (L.) Hitchc. var. divaricata (H; BMM)

Lasiacis nigra Davidse (H; BMM)

Lasiacis procerrima (Hackel) Hitchc. (H; BMM)

Lithachne pauciflora (Sw.) Beauv. ex Poiret (H; BMM)

Melinis minutiflora Beauv. (H; BPQ)

Muhlenbergia ciliata (Kunth) Kunth (H; BMM)

Muhlenbergia diversiglumis Trin. (H; BMM)

Muhlenbergia robusta (Fourn.) Hitchc. (H; BPQ)

Oplismenus hirtellus (L.) Beauv. var. hirtellus (H; BMM)

Panicum parviglume Hack. (H; BMM)

Panicum pulchellum Raddi (H; BMM)

Pseudechinolaena polystachya (Kunth) Stapf (H; BMM)

Zeugites americana Willd. var. mexicana (Kunth) McVaugh (H; BPQ)

Familia Smilacaceae

Smilax angustiflora DC. (V; BMM)

Smilax gymnopoda Apt (V; BMM)

Smilax lanceolata L. (V; BMM)

Smilax moranensis M. Martens \& Galeotti (V; BMM)

Smilcx subpubescens DC. (V; BMM)

\section{CLASE MAGNOLIOPSIDA}

\section{Familia Acanthaceae}

Barleria micans Nees (H; BMM)

Glockeria glandulosa Oerst. (H; BMM)

Justicia caudata A. Gray (H; BTC)

Ruellia eumorphantha Lindau (SS; BMM)

Ruellia hookeriana (Nees) Hemsl. (H; BTC)

Ruellia longituba D. Gibson (SS; BMM)

Ruellia matagalpae Lindau (H; BMM)

Ruellia petiolaris (Nees) T.F. Daniel (S; BMM, BPQ)

Ruellia stemonacanthoides (Oerst.) Hemsl. (H; BMM)

Familia Actinidiaceae

Saurauia comitis-rossei Schultes (T; BMM)

Saurauia conzattii Buscali (T; BMM)

Saurauia leucocarpa Schldl. (T; BMM)

Familia Amaranthaceae

Alternanthera lanceolata (Benth.) Schinz (SS; BMM) 
Iresine celosia $\mathrm{L}$. ( $\mathrm{H} ; \mathrm{BMM})$

Iresine grandis Standl. (S; BTC)

Familia Anacardiaceae

Pistacia mexicana Kunth (T; BTC)

Rhus radicans $\mathrm{L}$. var. radicans (S; BMM)

Rhus schiedeana Schldl. subsp. schiedeana (S; BMM)

Rhus terebinthifolia Schldl. (S; BMM, BPQ)

Familia Annonaceae

Rollinia mucosa (Jacq.) Baill. (T; BMM)

Familia Apiaceae

Apium leptophyllum (Pers.) F. Muell. ex Benth. (H; BMM, BPQ)

Familia Apocynaceae

Haplophyton cimicidum DC. (SS,S; BTC)

Haplophyton cinereum (A. Rich) Woodson (SS; BTC)

Thevetia ovata (Cav.) DC. (T; BTC)

Vallesia mexicana Muell. Arg. (T; BMM)

Familia Aquifoliaceae

Ilex brandegeana Loes. (T; BMM)

Familia Araliaceae

Dendropanax arboreus (L.) Decne. \& Planch. (S,T; BMM)

Oreopanax echinops (Schldl. \& Cham.) Decne. \& Planch. (S,T; BMM)

Oreopanax langlassei Standl. (T; BMM)

Oreopanax sanderianus Hemsl. (T; BMM)

Oreopanax xalapensis (Kunth) Decne. \& Planch. (S,T; BMM)

Familia Asclepiadaceae

Asclepias auriculata Kunth (H; BMM)

Asclepias circinalis (Decne.) Woodson (H; BMM,BPQ)

Asclepias laxiflora (Benth.) Decne. (H; BMM,BPQ)

Asclepias pellucida Fourn. (H; BMM)

Blepharodon mucronatum (Schldl.) Decne. (V; BTC)

Cynanchum astephanoides Standl. (V; BMM)

Cynanchum foetidum (Cav.) Kunth (V; BTC)

Gonolobus xanthotrichus Brandegee (V; BM)

Matelea rupestris (Brandegee) Woodson (V; BTC)

Familia Asteraceae

Acourtia carpholepis (Sch. Bip. ex A. Gray) Reveal \& King (H; BMM)

Ageratina arsenei (B.L. Rob.) King \& H. Rob. (H; BPQ)

Alloispermum integrifolium (DC.) H. Rob. (SS; BMM, BPQ)

Axiniphyllum scabrum (Zucc.) S.F. Blake (H; BMM)

Baccharis salicifolia (Ruiz \& Pav.) Pers. (S; BPQ)

Baccharis serrifolia DC. (S; BPQ)

Baccharis trinervis (Lam.) Pers. var. trinervis (S,SS; BPQ)

Bartlettina sordida (Less.) King \& H. Rob. (SS; BMM, BPQ)

Bartlettina tuerckheimii (Klatt) King \& H. Rob. (SS; BMM)

Brickellia glandulosa (Llave) McVaugh (S; BTC)

Brickellia veronicifolia (Kunth) A. Gray var. veronicifolia (S; BTC)

Chromolaena collina (DC.) King \& H. Rob. (S; BPQ)

Conyza sophiifolia Kunth (H; BTC)

Coreopsis mutica DC. var. carnosifolia D.J. Crawford (S; BPQ)

Critonia morifolia (Mill.) King \& H. Rob. (S,T; BMM)

Dahlia coccinea Cav. (H; BMM, BPQ)

Elephantopus mollis Kunth (H; BMM) 
Fleischmannia pycnocephala (Less.) King \& H. Rob. (H; BMM, BPQ)

Fleischmanniopsis leucocephala (Benth.) King \& H. Rob. (H; BMM, BPQ)

Gamochaeta americana (Mill.) Cabrera (H; BMM, BPQ)

Guardiola tulocarpus A. Gray (H; BMM, BPQ)

Heterotheca inuloides Cass. var. inuloides (H; BTC)

Koanophyllon galeottii (B.L. Rob.) King \& H. Rob. (S; BMM, BP)

Mikania cordifolia (L. f.) Willd. (V; BMM)

Mikania micrantha Kunth (V; BMM)

Montanoa standleyi V.A. Funk (SS; BMM)

Montanoa tomentosa Cerv. subsp. xanthiifolia (Sch. Bip.) V.A. Funk (S; BMM)

Piptothrix areolaris (DC.) King \& H. Rob. (S; BMM, BPQ)

Podachaenium eminens (Lag.) Sch. Bip. (S; BMM)

Pseudogynoxys chenopodioides (Kunth) Cabrera var. chenopodioides (V; BMM)

Rumfordia floribunda DC. var. australis R. W. Sanders (S; BMM)

Schistocarpha eupatorioides (Fenzl) Kuntze (H; BMM)

Senecio bracteatus Klatt (H; BPQ)

Sinclairia discolor (Humb. \& Bonpl.) Hemsl. (S; BMM)

Stevia subpubescens Lag. var. subpubescens (S; BMM)

Tagetes lucida Cav. (H; BTC)

Tithonia diversifolia (Hemsl.) A. Gray (H,S; BMM, BTC)

Trigonospermum melampodioides $\mathrm{DC}$. (H; BMM, BPQ)

Verbesina macvaughii B.L. Turner (S; BMM)

Vernonia deppeana Less. (S; BMM)

Vernonia karvinskiana DC. subsp. inuloides (DC.) S.B. Jones (S; BTC)

Vernonia karvinskiana DC. subsp. karvinskiana (S; BMM, BPQ)

Vernonia salvinae Hemsl. (SS; BMM)

Vernonia tortuosa (L.) S.F. Blake (S; BMM)

Viguiera microcephala Greenm. (SS; BMM, BPQ)

Youngia japonica (L.) DC. (H; BMM, BPQ)

\section{Familia Begoniaceae}

Begonia biserrata Lindl. (H; BMM)

Begonia boissieri DC. (H; BTC)

Begonia chivatoa Ziesenh. (H; BMM, BTC, BPQ)

Begonia fusca Liebm. (H; BMM, BPQ)

Begonia gracilis Kunth (H; BMM, BPQ)

Begonia pedata Liebm. (H; BMM)

Begonia squarrosa Liebm. (H; BMM)

\section{Familia Betulaceae}

Alnus acuminata Kunth subsp. arguta (Schldl.) Furlow (T; BMM, BPQ)

Carpinus caroliniana Walt. (T; BPQ)

\section{Familia Bignoniaceae}

Parmentiera edulis DC. (T; BTC)

Tecoma stans (L.) Kunth var. stans (S; BMM, BTC, BPQ)

Familia Boraginaceae

Cordia prunifolia I.M. Johnst. (T; BMM)

Cordia spinescens L. (H,S,V; BMM)

Heliotropium ternatum Vahl (S; BTC)

Lithospermum calcicola B.L. Rob. (H; BTC)

Tournefortia glabra L. (S; BMM)

Tournefortia trichocalycina DC. (SS; BTC)

Familia Buddlejaceae

Buddleja americana L. (S; BMM, BPQ)

Buddleja parviflora Kunth (S,T; BMM, BPQ) 


\section{Familia Burseraceae}

Bursera fagaroides (Kunth) Engl. var. fagaroides (S,T; BTC)

Bursera galeottiana Engl. (T; BTC)

Bursera schlechtendalii Engl. (T; BTC)

Bursera vejar-vazquesii Miranda (T; BTC)

\section{Familia Cactaceae}

Cephalocereus chrysacanthus (F.A.C. Weber) Britton \& Rose (H; BTC)

Ferocactus macrodiscus (C. Martius) Britton \& Rose var. macrodiscus (H; BTC)

Mammillaria collinsii (Britton \& Rose) Orcutt (H; BTC)

Mammillaria haageana Pfeiffer (H; BTC)

Mammillaria karwinskiana $\mathrm{C}$. Martius (H; BTC)

Neobuxbaumia mezcalensis (H. Brav.-Holl.) Backeb. (H; BTC)

Nopalea auberi (Pfeiffer) Salm-Dyck (H; BTC)

Nopalxochia ackermannii (Haworth) Kunth (H; BTC)

Rhipsalis baccifera (J. Miller) W. Stearn (E; BMM)

Stenocereus pruinosus (Otto) F. Buxb. (H; BTC)

\section{Familia Caesalpiniaceae}

Cercidium praecox (Ruiz \& Pav.) Harms (T,S; BTC)

Chamaecrista glandulosa (L.) Greene var. glandulosa (S; BMM)

Senna mollissima (Willd.) Irwin \& Barneby var. mollissima (T; BTC)

Senna pallida (Vahl) Irwin \& Barneby (S; BMM)

\section{Familia Campanulaceae}

Diastatea micrantha (Kunth) McVaugh (H; BMM, BPQ)

Lobelia aguana Kunth (SS; BPQ)

Lobelia laxiflora Kunth var. angustifolia DC. (H; BMM, BPQ)

\section{Familia Capparaceae}

Capparis heydeana Donn. Sm. (T; BMM)

Polanisia uniglandulosa (Cav.) DC. (H; BTC)

\section{Familia Caprifoliaceae}

Viburnum elatum Benth. (S,T; BMM)

Viburnum stenocalyx (Oerst.) Hemsl. (S,T; BMM)

Familia Caryophyllaceae

Arenaria alsinoides Willd. (H; BMM, BPQ)

Arenaria lanuginosa (Michx.) Rohrb. subsp. lanuginosa (H; BPQ)

Drymaria gracilis Cham. \& Schldl. subsp. gracilis (H; BMM)

\section{Familia Celastraceae}

Celastrus pringlei Rose (V; BMM)

Rhacoma tonduzii (Loes.) Standl. \& Steyerm. (T; BMM)

Zinowiewia integerrima (Turcz.) Turcz. (T; BPQ)

Familia Chloranthaceae

Hedyosmum mexicanum Cordemoy (S,T; BMM)

Familia Cistaceae

Helianthemum concolor (Riley) González Ortega (SS; BMM)

Familia Clethraceae

Clethra mexicana DC. (T; BMM)

Familia Clusiaceae

Clusia massoniana Lundell (T; BMM)

Clusia salvinii Donn. Sm. (T; BMM)

Hypericum uliginosum Kunth (SS; BPQ)

Vismia camparaguey Sprague \& Riley (T; BMM)

Vismia mexicana Schldl. (T; BMM) 


\section{Familia Convolvulaceae}

Evolvulus alsinoides L. var. alsinoides (V; BTC)

Ipomoea indica (Burm.) Merr. (V; BPQ)

Ipomoea mairetii Choisy (V; BMM)

Ipomoea purpurea (L.) Roth (H; BMM)

Ipomoea sagittata $\mathrm{Lam}$. (SS; BPQ)

Ipomoea signata House (V; BPQ)

Ipomoea suffulta (Kunth) G. Don (H, BTC)

Ipomoea trifida (Kunth) G. Don (V; BPQ)

Familia Cornaceae

Cornus disciflora DC. (S,T; BMM)

Familia Crassulaceae

Echeveria rosea $\mathrm{Lindl}$ ( $\mathrm{H}$; BMM)

Sedum chiapensis Rose ex Von Poellnitz (H; BMM)

Villadia nelsonii Rose (H; BMM)

\section{Familia Cucurbitaceae}

Melothria pendula L. (H,V; BMM, BPQ)

Sechium edule (Jacq.) Sw. (V; BPQ)

\section{Familia Ebenaceae}

Diospyros campechiana Lundell (T; BMM)

\section{Familia Elaeocarpaceae}

Muntingia calabura L. (T, BMM)

\section{Familia Ericaceae}

Agarista mexicana (Hemsl.) Judd var. mexicana (S; BPQ)

Arbutus xalapensis Kunth (T; BMM)

Arctostaphylos lucida (Small) Standl. (S; BMM, BPQ)

Arctostaphylos pyrifolia (Donn. Sm.) Standl. \& Steyerm. (S; BPQ)

Bejaria aestuans Mutis ex L. (S; BPQ)

Bejaria mexicana Benth. (S; BPQ)

Gaultheria odorata Willd. (S; BMM, BPQ)

Lyonia squamulosa M. Martens \& Galeotti. (S; BPQ)

Vaccinium confertum Kunth (S; BMM, BPQ)

Familia Erythroxylaceae

Erythroxylum pallidum Rose (S; BMM)

\section{Familia Euphorbiaceae}

Acalypha frederici Muell. Arg. (H; BMM)

Acalypha microcephala Muell. Arg. (SS; BMM)

Acalypha oligodonta Muell. Arg. (H; BMM)

Croton ciliato-glanduliferus Ortega (S; BMM)

Croton cortesianus Kunth (S; BTC)

Croton draco Schldl. var. panamensis Webster (T; BMM)

Euphorbia ariensis Kunth (H; BMM)

Euphorbia fulgens Karw. ex Klatt (SS; BMM, BPQ)

Euphorbia oerstediana (Klatt) Boiss. (H; BPQ)

Euphorbia pulcherrima L. (S; BTC)

Euphorbia whitei L.C. Wheeler (H,SS; BMM)

Phyllanthus lathyroides Kunth (H; BMM)

Phyllanthus niruri L. (H; BMM)

Ricinus communis L. (H,S; BMM, BPQ)

Tragia volubilis $\mathrm{L}$. (V; BMM, BTC)

\section{Familia Fabaceae}

Astragalus guatemalensis Hemsl. var. brevidentatus (Hemsl.) Barneby (H; BMM)

Canavalia hirsuta (M. Martens \& Galeotti) Standl. (V; BMM, BPQ) 
Clitoria polystachya Benth. (V; BMM)

Cologania biloba (Lindley) Nicholson (V; BMM)

Cologania broussonetti (Balb.) DC. (S; BPQ)

Coursetia caribaea (Jacq.) Lavin var. caribaea (H,SS,S; BTC)

Crotalaria acapulcensis Hook. \& Arn. (H; BPQ)

Crotalaria bupleurifolia Schldl. \& Cham. (H; BMM)

Crotalaria longirostrata Hook. \& Arn. (H; BMM)

Crotalaria mollicula Kunth (H; BMM, BPQ)

Crotalaria quercetorum Brandegee (H; BMM)

Crotalaria rotundifolia (Walter) Poiret var. vulgaris Windler (H; BMM)

Crotalaria sagittalis $\mathrm{L}$. (H; BMM)

Dalea leucostachys A. Gray var. eysenhardtioides (Hemsl.) Barneby (H; BPQ)

Desmodium amplifolium Hemsl. (S; BMM)

Desmodium helleri Peyr. (S; BMM, BPQ)

Desmodium intortum (Mill.) Urban (S; BMM)

Desmodium jaliscanum $\mathrm{S}$. Watson (S; BMM)

Desmodium molliculum (Kunth) DC. (H; BMM)

Diphysa puberulenta Rydb. (S; BPQ)

Erythrina americana Mill. (T; BMM, BTC)

Erythrina mexicana Krukoff (T; BMM)

Galactia incana (Rose) Standl. (SS; BMM, BPQ)

Indigofera miniata Ortega (SS; BMM, BPQ)

Indigofera thibaudiana DC. (S; BPQ)

Marina scopa Barneby (S; BMM)

Ormosia oaxacana Rudd (T; BMM)

Phaseolus chiapasanus Piper (V; BMM)

Phaseolus coccineus L. subsp. formosus (Kunth) Mari., Mesch. \& Stain. (H,V; BMM, BPQ)

Phaseolus lunatus L. var. sylvester Baud. (V; BPQ)

Rhynchosia longeracemosa M. Martens \& Galeotti (V; BMM, BPQ)

Stylosanthes guianensis $\mathrm{Sw}$. (H; BPQ)

Stylosanthes hamata (L.) Taub. (H; BPQ)

Stylosanthes humilis Kunth (H; BPQ)

Stylosanthes viscosa $\mathrm{Sw}$. (H; BPQ)

Tephrosia lanata M. Martens \& Galeotti. (S; BMM)

Tephrosia langlassei Micheli (S; BMM)

Zornia thymifolia Kunth (H; BMM, BPQ)

\section{Familia Fagaceae}

Quercus acutifolia Nee (T; BMM)

Quercus candicans Nee (T; BPQ)

Quercus cortesii Liebm. (T; BMM)

Quercus elliptica Nee (T; BMM, BPQ)

Quercus glaucoides M. Martens \& Galeotti. (T; BTC)

Quercus laurina Humb. \& Bonpl. (T; BMM, BPQ)

Quercus magnoliifolia Nee (T; BMM, BPQ, BTC)

Quercus martinezii C.H. Muller (T; BMM, BPQ)

Quercus rugosa Neé (S,T; BPQ)

Quercus scytophylla Liebm. (T; BMM, BPQ)

Quercus segoviensis Trel. (T; BPQ)

Quercus skinnerii Benth. (T; BPQ)

Quercus splendens Nee (T; BPQ, BTC)

Quercus urbanii Tiel. (T; BTC)

Quercus vicentensis Trel. (T; BMM)

Quercus xalapensis Humb. \& Bonpl. (T; BMM)

Familia Flacourtiaceae

Xylosma celsatrinum (Kunth) Standl. (S; BMM)

Xylosma flexuosum (Kunth) Hemsl. (T; BMM) 
Familia Gentianaceae

Centaurium brachycalyx Standl. \& L.O. Williams (H; BPQ)

Familia Geraniaceae

Geranium lilacinum Kunth (H; BPQ)

Familia Gesneriaceae

Achimenes antirrhina (DC.) Morton (H; BMM)

Achimenes glabrata (Zucc.) Fritsch. (H; BMM)

Columnea matudae (Wiehler) G. Kvist \& L. Skog (H; BMM)

Columnea purpusii Standl. (E; BMM)

Eucodonia andrieuxii (DC.) Wiehler (H; BTC)

Moussonia deppeana (Schldl. \& Cham.) Hanst. (SS; BMM)

Solenophora toucana D. Denham \& D. Gibson (S; BMM)

Familia Hydrophyllaceae

Wigandia urens (Ruiz \& Pav.) Kunth var. urens (S; BPQ)

Familia Lamiaceae

Cunila pycnantha Rob. \& Greenm. (H; BMM)

Hyptis oblongifolia Benth. (S; BMM, BPQ)

Hyptis pectinata (L.) Poir. (SS; BPQ)

Salvia adenophora Fern. (S; BPQ)

Salvia cinnabarina M. Martens \& Galeotti. (H; BMM, BPQ)

Salvia glabra M. Martens \& Galeotti. (SS; BMM)

Salvia inconspicua Benth. (H; BMM, BPQ)

Salvia karwinskii Benth. (SS; BMM)

Salvia langlassei Fern. (H; BMM)

Salvia lavanduloides Kunth (H,SS; BMM, BPQ)

Salvia littae Fernald (S; BPQ)

Salvia mexicana L. var. mexicana (H,SS,S; BPQ)

Salvia nicolsoniana Ramamoorthy (SS; BMM)

Salvia nitida Benth. (H; BMM, BPQ)

Salvia pubescens Benth. (H; BTC)

Salvia purpurea Cav. (H; BMM, BPQ)

Salvia semiatrata Zucc. (S; BPQ)

Salvia vitifolia Benth. (H; BMM)

Satureja macrostema (Benth.) Briq. var. laevigata (Standl.) McVaugh \& Schmid. (S; BPQ)

Stachys boraginoides Cham. \& Schldl. (H; BMM, BPQ)

Familia Lauraceae

Licaria capitata (Schldl. \& Cham.) Kostermans (T; BMM)

Litsea glaucescens Kunth (S,T; BMM)

Ocotea helicterifolia (Meissn.) Hemsl. (T; BMM)

Persea americana Mill. var. americana (T, BMM)

Familia Lentibulariaceae

Pinguicula heterophylla Benth. (H; BMM)

Pinguicula moranensis Kunth (H; BPQ)

Familia Loasaceae

Mentzelia hispida Willd. (H,SS; BTC)

Familia Loranthaceae

Psittacanthus macrantherus Eichler (HP; BPQ)

Psittacanthus ramiflorus (DC.) Don (HP; BMM, BPQ)

Struthanthus deppeanus (Schldl. \& Cham.) Trel. (HP; BPQ)

Struthanthus loniceroides (Van Tieghem) Kuijt (HP; BMM, BPQ)

Familia Lythraceae

Cuphea aequipetala Cav. (H,SS; BPQ) 
Cuphea appendiculata Benth. var. appendiculata (H; BMM, BPQ)

Cuphea cyanea DC. (H,SS; BMM)

Cuphea dibrachiata S. Graham (H; BMM)

Cuphea flavovirens S. Graham (H; BMM)

Cuphea hookeriana Walp. (H; BPQ)

Cuphea nudicostata Hemsl. (S; BMM)

Lythrum vulneraria Schrank (H,SS; BTC)

Familia Malpighiaceae

Bunchosia lanceolata Turcz. (S; BMM)

Byrsonima crassifolia (L.) Kunth (T; BMM, BPQ)

Galphimia glauca Cav. (S; BTC)

Heteropteris laurifolia (L.) A. Juss. (S,V; BMM)

Malpighia glabra L. (S,T; BMM)

Malpighia mexicana A. Juss. (S; BTC)

Familia Malvaceae

Malvaviscus arboreus Cav. var. mexicanus Schldl. (S; BMM)

Malvaviscus lanceolatus Rose (S,T; BMM, BPQ)

Sida haenkeana K. Presl (SS,S; BMM)

Sida rhombifolia L. (H,SS; BMM)

Familia Marcgraviaceae

Souroubea exauriculata Delpino (V; BMM)

Familia Melastomataceae

Arthrostema ciliatum Ruiz \& Pav. (H; BMM)

Clidemia capitellata (Bonpl.) D. Don (S; BMM)

Clidemia matudae L.O. Williams (S; BMM, BPQ)

Conostegia mexicana Cogn. (H; BMM)

Conostegia xalapensis (Kunth) G. Don ex DC. (S,T; BMM)

Heterocentron alatum Rose \& Standl. (H,SS; BMM)

Heterocentron subtriplinervium (Link. \& Otto) A. Br. \& Bouche (SS; BMM)

Leandra subseriata (Naudin) Cogn. (S; BMM, BPQ)

Miconia dodecandra (Desf.) Cogn. (S; BMM)

Miconia glaberrima (Schldl.) Naudin (S; BPQ)

Miconia sylvatica (Schldl.) Naud. (S; BMM, BPQ)

Monochaetum floribundum (Schldl.) Naudin (S; BMM)

Tibouchina naudiniana (Decne.) Cogn. (H; BMM)

Tibouchina scabriuscula (Schldl.) Cogn. (S; BMM)

Topobea maurofernandeziana Cogn. (T; BMM)

Familia Meliaceae

Guarea glabra Vahl (T; BMM)

Trichilia havanensis Jacq. (S,T; BMM)

Familia Menispermaceae

Cissampelos pareira L. (V; BMM)

Cissampelos tropaeolifolia DC. (V; BMM)

Familia Mimosaceae

Abarema zolleriana (Standl. \& Steyerm.) L. Rico (T; BMM)

Acacia angustissima (Mill.) Kuntze var. angustissima (S,T; BPQ)

Acacia pennatula (Schldl. \& Cham.) Benth. (S,T; BPQ)

Acacia velvae L. Rico (S; BPQ)

Calliandra eriophylla Benth. (S; BTC)

Calliandra grandiflora (L'Her.) Benth. (S; BPQ)

Calliandra hirsuta (G. Don) Benth. (S; BPQ)

Calliandra houstoniana (Mill.) Kuntze (S; BPQ)

Inga eriocarpa Benth. (T; BMM) 
Inga hintonii Sandw.(T; BMM)

Inga oerstediana Benth. ex Seemann (T; BMM)

Lysiloma auritum (Schldl.) Benth. (T; BPQ)

Lysiloma divaricata (Jacq.) Macbr. (T; BTC)

Mimosa albida Humb. \& Bonpl. ex Willd. var. albida (S; BPQ)

Zapoteca formosa (Kunth) H. Hern. subsp. formosa (S,T; BTC)

Zapoteca media (M. Martens \& Galeotti.) H. Hern. (S; BTC)

Zapoteca portoricensis (Jacq.) H. Hern. subsp. portoricensis (S ;BMM)

Zapoteca sousae H. Hern. \& A. Campos (S; BMM)

Familia Monimiaceae

Mollinedia viridiflora Tulasne (T; BMM)

Siparuna andina (Tulasne) DC. (S,T; BMM)

Familia Monotropaceae

Monotropa uniflora L. (H; BPQ)

Familia Moraceae

Dorstenia contrajerba L. var. contrajerba (H; BMM)

Dorstenia contrajerba L. var. tenuiloba (S.F. Blake) Standl. (H; BMM)

Ficus padifolia Kunth (T; BTC)

Ficus velutina Kunth ex Willd. (T; BMM)

Familia Myricaceae

Myrica cerifera L. (S,T; BMM, BPQ)

Myrica mexicana Willd. (S; BMM)

Familia Myrsinaceae

Ardisia compressa Kunth (T; BMM)

Ardisia escuintlensis Lundell (T; BMM)

Ardisia sexpartita Lundell (T; BMM)

Ardisia verapazensis Donn. Sm. (T; BMM)

Myrsine jurgensenii (Mez) Lundell (T; BMM)

Parathesis brevipes Lundell (T; BMM)

Parathesis chiapensis Fernald (T; BMM)

Parathesis columnaris Lundell (T; BMM)

Parathesis macronema Bullock (S,T; BMM)

Parathesis vulgata Lundell (S; BMM)

Rapanea ferruginea (Ruiz \& Pav.) Mez (S,T; BMM)

Familia Myrtaceae

Eugenia rekoi Standl. (S; BMM)

Eugenia rhombea (O. Berg.) Krug. \& Urb. ex Urb. (S,T; BMM)

Myrcia splendens (Sw.) DC. (T; BMM)

Myrcianthes fragans (Sw.) McVaugh (T; BMM)

Myrciaria floribunda (West.) O. Berg. (T; BMM)

Psidium guajava L. (S; BMM)

Familia Nyctaginaceae

Mirabilis sanguinea Heimer. (H; BTC)

Mirabilis violacea (L.) Heimerl (H; BTC)

Mirabilis viscosa Cav. (H; BTC)

Familia Onagraceae

Fuchsia arborescens Sims. (S; BMM)

Fuchsia chiapensis Brandegee (S; BMM, BPQ)

Fuchsia microphylla Kunth subsp. microphylla (S; BMM, BPQ)

Fuchsia paniculata Lindl. (S; BMM)

Fuchsia thymifolia Kunth subsp. thymifolia (S; BMM, BPQ)

Oenothera pubescens Willd. ex Spreng. (H; BPQ) 
Familia Orobanchaceae

Conopholis alpina Liebm. var. alpina (H; BTC)

Familia Passifloraceae

Passiflora cookii Killip (V; BMM)

Passiflora coriacea Juss. (V; BMM)

Passiflora membranacea Benth. (V; BMM)

Passiflora oaxacensis J. MacDougal (V; BMM)

Familia Phytolaccaceae

Phytolacca rugosa Kunth (H; BPQ)

\section{Familia Piperaceae}

Peperomia aggravescens Trel. (E; BMM)

Peperomia blanda (Jacq.) Kunth (E; BMM)

Peperomia coarctata Trel. \& Standl. (E; BMM)

Peperomia cobana DC. (E; BPQ)

Peperomia collocota Trel. (E; BMM))

Peperomia crassiuscula Millsp. (E; BMM, BPQ)

Peperomia deppeana Schldl. \& Cham. (E; BMM)

Peperomia galioides Kunth (E; BMM)

Peperomia granulosa Trel. (E; BMM)

Peperomia guatemalensis DC. (H; BMM)

Peperomia heterodoxa Standl. \& Steyerm. (E; BMM)

Peperomia mexicana Miq. (H; BMM, BPQ)

Peperomia nigropunctata Miq. (E; BMM)

Peperomia obtusifolia (L.) A. Dietr. (E; BMM, BPQ)

Peperomia peltata DC. (H; BMM, BTC)

Peperomia quadrifolia (L.) Kunth (E,H; BMM, BPQ)

Peperomia tacticana Trel. \& Standl. (H; BMM)

Piper aduncum L. (S; BMM, BTC)

Piper amalago L. (T; BTC)

Piper auritum Kunth (S,T BMM)

Piper diandrum DC. (S; BMM, BPQ)

Piper fallens Trel. (S; BMM)

Piper jacquemontianum Kunth (S; BMM, BPQ)

Piper luxii DC. (S; BMM)

Piper martensianum DC. (S; BMM)

Piper minarum Standl. \& Steyerm. (S; BMM)

Piper patzulinum Trel. \& Standl. (S; BMM)

Piper pogonioneuron Trel. \& Standl. (S; BMM)

Piper sancti-felicis Trel. (S; BTC)

Piper scabrum Sw. (S; BMM, BTC)

Piper schippianum Trel. \& Standl. (S; BMM, BPQ)

Piper stillans Trel. \& Standl. (S; BMM, BPQ)

Piper tacticanum Trel. \& Standl. (S; BMM)

Piper tecutlanum Trel. \& Standl. (S, BMM, BPQ)

Piper umbellatum L. (S; BMM)

Piper variable C. DC. ex Donn. Sm. (S; BMM)

Piper xanthostachyum DC. (S; BMM)

Piper yzabalanum C. DC. ex Donn. Sm. (S; BMM)

Familia Plantaginaceae

Plantago australis Lam. subsp. hirtella (Kunth) Rahn (H; BPQ)

Familia Polygalaceae

Monnina xalapensis Kunth (S,T; BPQ)

Polygala floribunda Benth. (S; BMM)

Polygala oaxacana Chodat (T; BTC) 
Polygala oophylla S.F. Blake (H; BPQ)

\section{Familia Proteaceae}

Roupala montana Aubl. (T; BMM)

Familia Pyrolaceae

Chimaphila maculata (L.) Pursh subsp. maculata (H; BPQ)

Familia Ranunculaceae

Clematis dioica L. (V; BTC)

Delphinium pedatisectum Hemsl. (H; BPQ)

\section{Familia Rhamnaceae}

Colubrina ehrenbergii Schldl. (T; BTC)

Gouania lupuloides (L.) Urban (V; BTC)

\section{Familia Rosaceae}

Amelanchier denticulata (Kunth) Koch var. denticulata (S,T; BTC)

Holodiscus argenteus (L. f.) Maxim. (S; BPQ)

Prunus cortapico Kerner (T; BMM)

Prunus rhamnoides Koehne (T; BMM)

Prunus serotina Ehrh. subsp. capuli (Cav.) McVaugh (T; BMM)

Rubus trichomallus Schldl. (S; BMM, BPQ)

Rubus verae-crucis Rydb. (S; BMM, BPQ)

\section{Familia Rubiaceae}

Borreria laevis (Lam.) Griseb. (H; BMM, BPQ)

Bouvardia cordifolia DC. (S; BMM)

Chiococca alba (L.) Hitchc. (S,V; BMM)

Chiococca phaenostemon Schldl. (S; BMM)

Coccocypselum guianense (Aubl.) Schummach. (H; BMM)

Coffea arabica L. (S; BMM)

Crusea calocephala DC. (H; BMM)

Crusea coccinea DC. var. breviloba Loes. (H; BMM, BPQ)

Deppea hintonii Bullock (S; BMM)

Deppea miahuatlana Lorence (S; BMM)

Deppea pubescens Hemsl. (S; BMM)

Didymaea alsinoides (Schldl. \& Cham.) Standl. (H; BMM)

Galium aschenbornii Schauer (H; BMM)

Galium mexicanum Kunth subsp. asperrimum (A. Gray) Dampster (H; BMM, BPQ)

Gonzalagunia panamensis (Cav.) K. Schum. (SS; BMM)

Guettarda macrosperma Donn. Sm. (H; BTC)

Hoffmannia cryptoneura Standl. (S; BMM)

Hoffmannia oaxacensis Dwyer (S; BMM)

Omiltemia longipes Standl. (SS; BMM)

Palicourea padifolia (Willd. ex Roem. \& Schult.) Taylor \& Lorence (S; BMM)

Posoqueria coriacea M. Martens \& Galeotti. (T; BMM)

Posoqueria latifolia (Rudge.) Ruiz \& Pav. (T; BMM)

Psychotria graciliflora (Benth. ex Oerst.) Hemsl. (S; BMM)

Psychotria panamensis Standl. var. panamensis (S; BMM)

Psychotria pubescens Sw. (S; BMM)

Psychotria trichotoma M. Martens \& Galeotti. (S; BMM)

Randia cookii Standl. (S; BMM)

Rondeletia albida Lundell (S; BMM)

Rondeletia buddleioides Benth. (S,T; BMM)

Rondeletia jurgensenii Hemsl. (S; BMM)

Sommera arborescens Schldl. (T; BMM)

Sommera grandis (Bartl.) Standl. (T; BMM) 


\section{Familia Sabiaceae}

Meliosma oaxacana Standl. (T; BMM)

Familia Salicaceae

Salix bonplandiana Kunth var. bonplandiana (T; BTC)

Familia Sapindaceae

Dodonaea viscosa (L.) Jacq. (S,T; BPQ)

Paullinia clavigera Schldl. (V; BMM)

Paullinia costaricensis Radlk. (V; BPQ)

Paullinia pinnata L. (V; BMM)

Sapindus saponaria $\mathrm{L}$. (T; BTC)

Serjania sordida Radlk. (V; BMM)

Familia Scrophulariaceae

Alonsoa caulinata Ruiz \& Pav. (H; BMM)

Calceolaria mexicana Benth. (H; BMM)

Castilleja lithospermoides Kunth (H; BMM)

Lamourouxia multifida Kunth (H; BPQ)

Lamourouxia xalapensis Kunth (H,S; BPQ)

Leucocarpus perfoliatus (Kunth) Benth. (H,SS; BMM)

Lophospermum hintonii Elisens (V; BMM)

Penstemon isophyllus Benth. (H; BPQ)

Russelia coccinea (L.) Wetts. (H,SS; BMM)

Russelia ternifolia Kunth (H; BMM)

Familia Simaroubaceae

Picramnia antidesma Swartz (S,T; BMM)

Picramnia lindeniana Tulasne (S; BMM)

Picramnia locuples Standl. (S; BMM)

Recchia connaroides (Loes. \& Solr.) Standl. (T; BTC)

\section{Familia Solanaceae}

Brachistus stramonifolius (Kunth) Miers (H; BMM)

Capsicum rhomboideum (Dunal) Kuntze (S; BTC)

Cestrum glanduliferum Francey (S; BMM)

Cestrum megalophyllum Dunal (T; BMM)

Physalis caudella Standl. (H; BMM)

Physalis sancti-josephi Dunal (H; BTC)

Solandra grandiflora $\mathrm{Sw}$. (S; BPQ)

Solanum acerifolium Dunal (S; BMM, BPQ)

Solanum aphyodendron S. Knapp (S; BMM, BPQ)

Solanum appendiculatum Humb. \& Bonpl. ex Dunal (V; BMM)

Solanum chrysotrichum Schldl. (S; BMM, BPQ)

Solanum nigrescens $\mathrm{M}$. Martens \& Galeotti. (V; BPQ)

Solanum wendlandii Hook. f. (V; BPQ)

\section{Familia Staphyleaceae}

Turpinia occidentalis (Sw.) G. Don subsp. breviflora Croat (S,T; BMM)

Turpinia occidentalis (Sw.) G. Don subsp. occidentalis (S,T; BMM)

Familia Sterculiaceae

Ayenia manzanilloa Rose (S; BTC)

Chiranthodendron pentadactylon Larreategui (T; BMM)

Familia Styracaceae

Styrax polyneurus Perkins (S; BTC)

Styrax ramirezii Greenm. (T; BMM)

Familia Symplocaceae

Symplocos limoncillo Humb. \& Bonpl. (T; BMM)

Symplocos pycnantha Hemsl. (T; BMM) 


\section{Familia Theaceae}

Cleyera theoides (Sw.) Choisy (T; BMM)

Ternstroemia lineata DC. subsp. lineata (T; BMM, BPQ)

Familia Thymelaeaceae

Daphnopsis nevlingii J. Ram. \& Contreras-Jimenez (S,T; BMM)

\section{Familia Tiliaceae}

Heliocarpus americanus L. (S; BTC)

Triumfetta dehiscens Rose (S; BMM)

Triumfetta grandiflora Vahl (S; BPQ)

Triumfetta semitriloba Jacq. (S,T; BMM)

Triumfetta speciosa Seem. (S; BMM, BPQ)

\section{Familia Turneraceae}

Turnera diffusa Willd. (S; BTC)

\section{Familia Ulmaceae}

Trema micrantha (L.) Blume var. micrantha (S,T; BMM)

Ulmus mexicana (Liebm.) Planch. (T; BMM)

\section{Familia Urticaceae}

Boehmeria caudata Swartz (S; BMM)

Boehmeria nivea (L.) Gaudin (H,SS; BMM)

Boehmeria ulmifolia Wedd. (S; BPQ)

Myriocarpa obovata Donn. Sm. (T; BMM)

Phenax hirtus (Sw.) Wedd. var. hirtus (H,SS,S; BMM)

Pilea pubescens Liebm. (H; BMM)

Pouzolzia nivea S. Watson (S; BMM)

Urera caracasana (Jacq.) Griseb. (S; BMM)

\section{Familia Valerianaceae}

Valeriana scandens L. var. scandens (V; BMM)

Valeriana sorbifolia Kunth var. sorbifolia (H; BMM)

\section{Familia Verbenaceae}

Citharexylum donnell-smithii Greenm. var. pubescens Moldenke (S,T; BMM)

Lantana hispida Kunth (S; BPQ)

\section{Familia Violaceae}

Viola humilis Kunth (H; BPQ)

Familia Vitaceae

Vitis cinerea Engelm. (V; BMM)

Vitis tiliifolia Kunth (V, BMM) 